\title{
How Suboptimal are Linear Sharing Rules?
}

\author{
Jensen, Bjarne Astrup ; Nielsen, Jørgen Aase
}

Document Version

Accepted author manuscript

Published in:

Annals of Finance

DOI:

10.1007/s10436-016-0279-3

Publication date:

2016

License

Unspecified

Citation for published version (APA):

Jensen, B. A., \& Nielsen, J. A. (2016). How Suboptimal are Linear Sharing Rules? Annals of Finance, 12(2), 221-243. https://doi.org/10.1007/s10436-016-0279-3

Link to publication in CBS Research Portal

\section{General rights}

Copyright and moral rights for the publications made accessible in the public portal are retained by the authors and/or other copyright owners and it is a condition of accessing publications that users recognise and abide by the legal requirements associated with these rights.

Take down policy

If you believe that this document breaches copyright please contact us (research.lib@cbs.dk) providing details, and we will remove access to the work immediately and investigate your claim. 


\title{
How Suboptimal Are Linear Sharing Rules?
}

\author{
Bjarne Astrup Jensen and Jorgen Aase Nielsen
}

Journal article (Post print version)

Cite: How Suboptimal Are Linear Sharing Rules?. / Astrup Jensen, Bjarne; Nielsen, Jargen Aase. In: Annals of Finance, Vol. 12, No. 2, 2016, p. 221-243.

The final publication is available at Springer via http://dx.doi.org/10.1007/s10436-016-0279-3

Uploaded to Research@CBS: September 2016 


\title{
How suboptimal are linear sharing rules?
}

\author{
Bjarne Astrup Jensen • Jørgen Aase Nielsen
}

April 15, 2016

\begin{abstract}
The objective of this paper is to analyze criteria for portfolio choice when two investors are forced to invest in a common portfolio and share the proceeds by a linear sharing rule. A similar situation with many investors is typical for defined contribution pension schemes. The restriction implies two sources of suboptimal investment decisions as seen from each of the two investors individually. One is the suboptimal choice of portfolio, the other is the forced linear sharing rule. We measure the combined consequence for each investor by their respective loss in wealth equivalent. We show that significant losses can arise when investors are diverse in their risk attitude. We also show that an investor with a low degree of risk aversion, like the logarithmic or the square root investor, often applied in portfolio choice models, can either inflict or be subject to severe losses when being forced to participate in such a common investment pool.
\end{abstract}

JEL Classification Code: G11

Key Words: Constrained portfolio choice, Pareto optimal sharing rules, suboptimal sharing rules, linear sharing rules.

\section{Introduction}

A sharing rule is a commonly used term to describe how aggregate consumption is distributed among individuals in a general equilibrium or how the total return on an investment made by multiple investors in common is distributed among the individual investors.

Whenever investors leave the investment decision or some parts of it to an agent or engages in investment activities in common with other investors there is a risk that the result becomes suboptimal, as seen from the viewpoint of a well informed investor. Investors who lack complete information may find it worthwhile to encounter management fees in order to compensate for lack of information in order to acquire, e.g., expertise in portfolio management. However, there is also a risk that the investor encounters costs of suboptimal dynamic asset allocation due to conflicting interests with the manager or other investors in a common pool.

There is a large literature analyzing various effects of constrained portfolio choice and incomplete information on portfolio decisions, and a thorough literature survey is beyond the scope of this paper. We limit ourselves to a few references that address these problems. Rogers (2001) analyzes two situations in a continuous time model with suboptimal investment policies relative to the benchmark of complete information and continuous portfolio revisions. One of the models imposes the restriction of only allowing discrete portfolio revisions, the other model

Bjarne Astrup Jensen

Department of Finance, Copenhagen Business School, Solbjerg Plads 3, 2000 Frederiksberg, Denmark, e-mail:ba.fi@cbs.dk, Phone: +45 38153614, Fax: +45 38153600.

Jørgen Aase Nielsen

Department of Mathematical Sciences, University of Aarhus, e-mail:atsjan@math.au.dk 
imposes a priori uncertainty about the rate of return. The finding is that the degree of suboptimality due to the restriction of discrete portfolio revisions is of minor importance, whereas the uncertainty about the rate of return can be substantial. In recent papers, Larsen and Munk (2012) address the issue of incomplete information and incomplete menu of assets in a rather general setting for a single investor with a focus on the importance of the hedging term for a stochastically varying investment opportunity set; and Flor and Larsen (2014) address the issue of ambiguity aversion when the investor does not know the true return distributions. We refer to the rather elaborate list of references in these papers for a broad variety of contributions dealing with various issues of time-varying risk premia and investment opportunity sets as well as incomplete information about return distributions.

The problem which we want to analyze in this paper - although in a simple and highly stylized manner - has to our knowledge not been addressed in the literature. We consider a model where we have multiple investors and one agent, and we only consider consumption at the final date. The investors are forced to invest in a common pool and the final wealth must be distributed to the investors in a linear fashion. An appropriate interpretation of this scenario of a suboptimal investment environment is the situation faced by many pension savers in asset backed, defined contribution pension schemes, where investors are forced by law or by labor market contracts to invest in a common portfolio and to share the aggregate result according to a linear sharing rule. Whenever investors differ in their attitude towards risk, this is different from what would have been the result if independent investors were allowed to find an unconstrained individual solution to their consumption and investment problem.

The suboptimality comes from three sources. The requirement of a common investment portfolio is one source of suboptimality; relatively more risk averse investors prefer a less risky portfolio than relatively less risk averse investors. Another source of suboptimality is the requirement of a linear sharing rule, which is usually only optimal in a situation where investors are up- or down-scaled copies of each other. In the terminology of financial economics each of these restrictions cause the market for financial assets to appear incomplete for each individual investor, even if the market is overall complete if investors were allowed to act in an unconstrained manner.

The third source of suboptimality is the fact that the manager, the investment officer in the pension fund, may not have complete information about the risk attitudes of the individual pension savers. We do not take this aspect into account in this paper, but assume that the manager is adequately informed about individual investor preferences.

It is sometimes argued that investors can circumvent investment decisions made within the common pool by adjusting their individual financial exposure. E.g., mandatory pension savers that disagree with their pension fund about the magnitude of exposure to risky assets can adjust for this through financial decisions of their own. However, this is not always possible. E.g., investors may find it difficult to short risky positions individually in case they find the common portfolio in the pension fund too risky. They may also find themselves borrowing restricted if the purpose of taking out additional loans is to increase their individual risky investments.

The agent in this paper is assumed to choose an investment strategy in accordance with criteria that do not influence his own reward. The issues of incentive compatible contracting and payment to the agent are kept outside our modeling framework.

Investors in an exchange economy are taking the market portfolio and the aggregate result as given. It means that the distribution of the aggregate future consumption is known. The role of the capital market is only to trade these future consumption opportunities across investors, whereas investments in production facilities that can make the future consumption an endogenous variable are outside the scope of the investors' opportunity set. In this paper the aggregate result for which a sharing rule must be established is endogenous, although only viewed upon in isolation without any considerations about general equilibrium properties.

It has been a longstanding tradition in financial economics to study the class of CRRA utility functions. Such preferences facilitate analytical solutions to individual portfolio choice problems and allows for straightforward aggregation properties in order to reach a Pareto optimal general equilibrium and the associated pricing kernel, where sharing rules are linear and in accordance with the initial wealth distribution. We follow this path as far as the individual preferences are concerned. In contrast to this, Pareto optimal sharing rules in a market equilibrium where investors are heterogeneous with respect to their relative risk aversion parameter are less straightforward to determine.

The seminal paper in this area is due to Dumas (1989), who established a modeling framework with two individuals, one with a logarithmic utility function and another with a power utility function. The results in Dumas (1989) 
are based on numerical solution techniques. Other subsequent papers along these lines are Benninga and Mayshar (2000), Cvitanić et al. (2012), Franke and Lüders (2010), Franke et al. (1998, 1999), Stapleton and Subrahmanyam (1990), Wang (1996) and Weinbaum (2009). All of these papers are within a framework of either an exchange economy or the CIR production economy model, cf. Cox et al. (1985), which assumes a production process that is stochastically homogeneous of degree one.

There is little guidance in the literature on how to choose the investment in a situation where investors can shape the risk profile of the aggregate consumption beyond a pure scale factor. The same is true for the situation where the sharing does not take place in a general equilibrium exchange economy context, but may be limited to a partial equilibrium where a particular group of investors for some reason are bound to invest in common. The latter is the viewpoint of the present analysis. We derive the constrained Pareto optimal terminal wealth for two investors from their individual preferences in a setting where the investors are forced to accept a linear sharing rule. We do this parametrically in the weighting between their utility functions with their individual preferences as boundary cases. We also derive how this terminal wealth varies with their relative risk aversion parameter to study the effects of diversity and compare the constrained Pareto optimal solution to situations with some ad hoc chosen investment criteria. The results of this paper necessarily rely on numerically calculated optimal solutions. Analytical results only exist for a special combination of parameters to be discussed in the following section 2 .

We measure the distance to a first best Pareto optimal sharing rule by calculating the wealth equivalents, i.e. the fraction of initial wealth that in a first best solution gives the same level of expected utility as the initial wealth invested in the constrained Pareto optimal manner. We find that for many cases with sufficient diversity among the investors the loss due to the constraints can be substantial and in the order of $10-20 \%$ or even more; we also find that for less pronounced diversity the forced common portfolio requirement and the linear sharing rule do not appear as severe restrictions. Furthermore, the loss in wealth equivalents is to a large extent driven by the extreme tails of the final wealth distribution.

The paper is organized as follows. Section 2 describes some relevant and both well known and less well known results from the literature on heterogeneous agents. Section 3 describes the financial market. Section 4 derives the unconstrained portfolio choice for an investor with a CRRA utility function (Theorem 1) and also the interrelationship between two such investors and their relative position with respect to their aggregate wealth (Theorem 2). Section 5 deals with aspects of the linear sharing requirement. Subsection 5.1 outlines an ad hoc decision rule, as a suggestion for an easily implementable investment criterion for the agent in order to compromise between two heterogeneous investors, given the linear sharing rule restriction. It is the portfolio policy for a single CRRA investor that imposes the same relative loss in both agents' wealth equivalents. Subsection 5.2 derives the constrained Pareto optimal investment behaviour numerically for a given utility weighting of the two investors. Subsection 5.3 extends the derivations in subsection 5.2 by letting the utility weighting be endogenously determined such that the relative losses in wealth equivalents are equally distributed among the investors. Furthermore, the numerical results are compared to the ad hoc criteria from subsection 5.1. In subsection 5.4 we show how the elasticity of the optimal final wealth with respect to the utility weighting varies across the outcomes. Section 6 summarizes and concludes the paper. Proofs and technical details are found in the Appendices.

\section{Known models for exchange economies}

The literature on Pareto optimal sharing rules in exchange economies with agents that are heterogeneous with respect to their degree of relative risk aversion contains a few general results. It is well known, cf. Benninga and Mayshar (2000), Dumas (1989) and Wang (1996), among others, that with two agents differing in their degree of risk aversion, the sharing rules are far from being linear. Instead, they are characterized by the following two properties:

- the (relatively) most risk averse investor receives a concave function of aggregate output, with a slope that ranges from one when aggregate output shrinks to zero and decreases towards zero when aggregate output goes to infinity

- the (relatively) least risk averse investor receives a convex function of aggregate output with a slope converging to zero when aggregate output shrinks to zero and increases towards one when aggregate output goes to infinity

Hence, the relatively least risk averse investor positions himself as an investor "taking bets" on the right tail of the distribution of aggregate consumption, whereas the relatively most risk averse investor protects himself relatively 
in the left tail of the distribution of aggregate consumption in return for leaving the bets on the right tail of the distribution to the relatively least risk averse investor.

When the two investors have CRRA utility functions with risk aversion parameters differing by a factor two, the sharing rules can be given in closed form. The same is possible for two investors or more when the risk aversion parameters deviate by multiples of three and/or four. Technically, the closed form solution relies on the ability to analytically solve for roots of polynomials up to the order four, as, e.g., demonstrated in Weinbaum (2009).

It has been shown that with more than two types of agents the ones "in between" receive an S-shaped function of aggregate output which is first concave and then convex after a reflection point. See Hara (2006) and Hara et al. (2007) for such results. These generalizations are still within the context of an exchange economy. To our knowledge this modeling framework has not yet been applied in a more general equilibrium setting, where the aggregate consumption and its risk profile is endogenously determined.

\section{The financial market}

The menu of financial assets used in this paper is a simple and standard one. Despite the simplicity, this setup is sufficiently rich to illuminate the properties of the economic problem and the suboptimality of the solution; and since the optimization problem we pose below must be solved by time-consuming numerical methods we find it reasonable to use as simple a setup as possible.

Investors are assumed to be able to invest in a combination of

1. a bank account with a constant rate of return $r$

2. a risky asset with a lognormal price process and a constant market price of risk $\eta$ :

$$
d P_{t}=(r+\eta \sigma) P_{t} d t+\sigma P_{t} d Z_{t},
$$

where the price process in (1) is specified under the real world probability measure and $Z_{t}$ is a standard Brownian motion.

The pricing kernel $M_{t}$ implied by the price process (1) is of the well-known log-normal type:

$$
M_{t}=e^{-r t-\frac{1}{2} \eta^{2} t-\eta Z_{t}} .
$$

The inverse of the pricing kernel $M_{t}^{-1}$ is known to be the value of the optimal growth portfolio chosen by an investor with a logarithmic utility function, $U\left(W_{T}\right) \equiv \log W_{T} \cdot{ }^{1}$

Individual investors take the price processes and the associated pricing kernel as given. These investors are also assumed to have common beliefs about the stochastic price process $P_{t}$. We do this because the setup is well known and renders simple analytical solutions for standard utility optimization problems for a single investor with these utility preferences. Such analytical solutions are useful in order to perform sensitivity analyses and can also be used to check the validity of the numerical calculations we are going to perform later on.

\section{Pareto optimal sharing rules: Two investors}

Consider an investor who solves the following expected utility problem with a CRRA utility function with relative risk aversion parameter parameter $\gamma$ :

$$
\begin{aligned}
& \operatorname{Max} E_{0}\left[U\left(W_{T}\right)\right]= \begin{cases}E_{0}\left[\frac{1}{1-\gamma} W_{T}^{1-\gamma}\right] & (\text { for } \gamma \neq 1) \\
E_{0}\left[\log W_{T}\right] & (\text { for } \gamma=1)\end{cases} \\
& \text { s.t. } \\
& E_{0}\left[M_{T} W_{T}\right]=W_{0} \quad[\lambda] .
\end{aligned}
$$

1 See Duffie (1996), chapter 6 or Merton (1992), chapter 6, among others for this standard result. 
The optimization problem in (3)-(4) is formulated in the standard way of the martingale method, which transforms the dynamic optimization problem into a static optimization problem. The Lagrangian multiplier $\lambda$ associated with the budget constraint in (4) is the shadow price of initial wealth.

The optimal portfolio choice is given in the following Theorem 1, which restates well-known results from the literature. The basic portfolio optimization model goes back to Merton (1971). As for the formulation in terms of the martingale method, the details are worked out in, e.g., Brennan and Torous (1999), Jensen and Sørensen (2001) and Balder and Mahayni (2010). We adopt the notation from the latter.

Theorem 1 (i) The optimal distribution of wealth at the horizon $T$ for any investor with

- a CRRA utility function

- a relative risk aversion parameter $\gamma$

- access to a complete market with the pricing kernel as given in (2)

is a log-normal distribution characterized in different ways in Equations (5)-(6) with $\pi \equiv \frac{\eta}{\gamma \sigma}$ being the constant fraction of wealth allocated to the risky asset:

$$
\begin{aligned}
& W_{T}=W_{0} e^{\left(r+\pi \eta \sigma-\frac{1}{2} \pi^{2} \sigma^{2}\right) T+\pi \sigma Z_{T}} \\
& W_{T}=W_{0}\left(P_{T} / P_{0}\right)^{\pi} e^{(1-\pi)\left(r+\frac{1}{2} \pi \sigma^{2}\right) T+\pi \sigma Z_{T}} .
\end{aligned}
$$

(ii) The optimal level of expected utility can - for $\gamma \neq 1-$ be written as

$$
J_{0}\left(W_{0} ; T, \gamma\right) \equiv E_{0}\left[\frac{W_{T}^{1-\gamma}}{1-\gamma}\right]=\frac{\left(W_{0} e^{\left(r+\frac{1}{2 \gamma} \eta^{2}\right) T}\right)^{1-\gamma}}{1-\gamma} .
$$

For $\gamma=1$ this becomes

$$
J_{0}\left(W_{0} ; T, 1\right)=\log W_{0}+r T+\frac{1}{2} \eta^{2} T
$$

The unconstrained sharing rule for two investors is stated in the following Theorem 2. It is automatically a Pareto-optimal sharing rule, since investors are trading in a complete and competitive market. The case with multiple investors with CRRA utility functions and identical relative risk aversion parameters is a work horse in the finance literature, because it allows aggregation from the individual level to the aggregate level in a straightforward manner: Any individual investor demands his fair share of the value of the aggregate portfolio.

Remark 1 Although the portfolio weight has the volatility $\sigma$ as an explicit parameter, it disappears in the expression for terminal wealth in Equation (5), which only contain the market risk premium $\eta$ (the "Sharpe ratio") and the relative risk aversion parameter $\gamma$ :

$$
\pi \sigma=\frac{\eta}{\gamma}, \quad \pi \sigma \eta=\frac{\eta^{2}}{\gamma}, \quad \pi^{2} \sigma^{2}=\frac{\eta^{2}}{\gamma^{2}} .
$$

This is so because the fundamental source of risk is the movement of the Brownian motion. The investor is interested in the trade off between the risk in the portfolio $(\pi \sigma)$ and the risk premium received $(\eta \sigma)$ as compensation for carrying this risk. The asset with price process $P_{t}$ is a specific carrier of this fundamental risk, but potentially infinitely many other assets could be chosen to make the market complete.

Theorem 2 Consider two investors having CRRA utility functions with relative risk aversion parameters $\gamma_{1}$ and $\gamma_{2}$, respectively, and facing a complete market with the pricing kernel as given in (2). Then the following is true:

(i) If the investors have identical values for $\gamma_{j}$, the Pareto optimal sharing rule can be written in terms of the aggregate portfolio:

$$
W_{j, T}=\frac{W_{j, 0}}{\mathcal{W}_{0}} \mathcal{W}_{T}
$$


where $\mathcal{W}_{t} \equiv W_{1, t}+W_{2, t}$ is the value of the aggregate portfolio at time $t$.

(ii) If the investors have different values for $\gamma_{j}$, the relative positioning of these two investors is given by:

$$
W_{i, T}=W_{i, 0} e^{r T} e^{\frac{1}{2}\left(1-\Gamma_{i}^{2}\right) \eta^{2} T+\frac{\eta}{\gamma_{i}} Z_{T}}, \quad i=1,2
$$

where $\Gamma_{i} \equiv 1-\frac{1}{\gamma_{i}}$.

The distribution of the individual investors' terminal wealth in terms of aggregate wealth becomes:

$$
\begin{aligned}
W_{1, T} & =\frac{\phi e^{\frac{1}{2}\left(\Gamma_{2}^{2}-\Gamma_{1}^{2}\right) \eta^{2} T+\left(\Gamma_{2}-\Gamma_{1}\right) \eta Z_{T}}}{1-\phi+\phi e^{\frac{1}{2}\left(\Gamma_{2}^{2}-\Gamma_{1}^{2}\right) \eta^{2} T+\left(\Gamma_{2}-\Gamma_{1}\right) \eta Z_{T}}} \mathcal{W}_{T} \\
W_{2, T} & =\frac{1-\phi}{1-\phi+\phi e^{\frac{1}{2}\left(\Gamma_{2}^{2}-\Gamma_{1}^{2}\right) \eta^{2} T+\left(\Gamma_{2}-\Gamma_{1}\right) \eta Z_{T}}} \mathcal{W}_{T} \\
\phi & \equiv \frac{W_{1,0}}{W_{1,0}+W_{2,0}} .
\end{aligned}
$$

Proof These relations are immediate consequences of relation (5) in Theorem 1.

Note that the distribution of aggregate wealth $\mathcal{W}_{T}$ for the case with identical values of $\gamma$ could be obtained as the solution to one optimization problem with initial wealth equal to the sum of the two individual initial wealth levels. The distribution among the agents is simple and linear in accordance with the initial wealth distribution.

In contrast to this, the aggregate wealth $\mathcal{W}_{T}$ for the case with different values of $\gamma$, i.e. in (ii), is no longer the result of one simple and immediately identifiable optimization problem. Rather, it is by definition equal to the sum of the results from two optimization problems. However, given that the outcome is Pareto optimal it is possible to identify such an optimization problem in terms of a representative investor that solves for the aggregate portfolio. But if this optimization problem was identified, it still remains to identify the sharing rule.

Remark 2 Consider the portfolio policy in (11)-(14). Low values of $Z_{T}$ correspond to high values of the pricing kernel and low values of the aggregate portfolio. Assume that investor 1 is the relatively most risk tolerant, i.e. $\Gamma_{2}>\Gamma_{1}$. Then investor 1 dominates the "high end" where $Z_{T}$ is diverging to $\infty$, whereas investor 2 dominates the "low end" where $Z_{T}$ is diverging to $-\infty$.

Remark 3 From the literature on heterogeneous agents ${ }^{2}$ it is well known that in an exchange economy context, where the aggregate "market portfolio" is exogenously given and $\gamma_{2}=2 \gamma_{1}$, the solution in (12)-(14) has the property that $W_{2, T}^{2}$ is proportional to $W_{1, T}$. This is indeed also the case here; it is a straightforward calculation to show that

$$
W_{2, T}^{2}=\left[\frac{W_{2,0}^{2}}{W_{1,0}} e^{\left(r+\left(\frac{\eta}{2 \gamma_{1}}\right)^{2}\right) T}\right] W_{1, T} .
$$

However, the distribution between the two investors as well as the aggregate investment strategy is endogenously derived in our setting and not the result of finding the sharing rule in an exchange economy. This is revealed, e.g., by looking at the behavior for "large" values of $\gamma_{1}$, and, hence, also for $\gamma_{2}$. There both investors prefer to invest almost everything in the bank account and only include a small amount of risk in the aggregate portfolio. In an exchange economy context investors are not able to affect aggregate risk; they are only able to find an allocation of the exogenously given risk between the investors.

\footnotetext{
2 See Benninga and Mayshar (2000), Dumas (1989), Wang (1996) and Weinbaum (2009), among others.
} 


\section{When linear sharing is required}

Assume now that investors are forced to invest in the same portfolio and to share the final result in a linear fashion. This leaves the manager of the pension fund with the problem of formulating criteria for the portfolio choice. Despite the fact that each participant has an individual preference for the investment strategy, the pension fund manager must make a common choice and distribute the proceeds in a linear fashion. This section describes a simple ad hoc suggestion as to the implementation of this portfolio choice.

We measure the utility loss by means of the wealth equivalent, which is the amount of initial wealth that, given the ability to invest optimally in an unconstrained way, gives the investor the same level of expected utility as one unit of wealth invested in a constrained way; i.e. under the restrictions of a common portfolio and a mandatory linear sharing rule. The benchmark for comparison is the result of an unconstrained expected utility maximization of end-of-period wealth.

We restrict the analysis to the case of two investors. We believe that the basic insight is sufficiently well demonstrated, and it allows us to depict the results graphically. Furthermore, the numerical solutions to be derived in subsection 5.3 are already quite demanding with just two investors.

\subsection{Choosing a compromise value of $\gamma$ ?}

One way to reach a compromise solution is to choose a value $\bar{\gamma}$ of the relative risk aversion parameter and implement the common investment strategy in the same way as an individual investor with a CRRA utility function with this risk aversion parameter would do. We have expressions for the portfolio choice in closed form; and the negative effect on each of the individual investors of being forced to participate in this common scheme, as expressed by the wealth equivalents, is known analytically as stated in Theorem $3 .^{3}$

Theorem 3 The wealth equivalent $\widehat{W}_{0}$ for an investor

- with a CRRA utility function with risk aversion parameter $\gamma$

- with initial wealth $W_{0}$ and investment horizon $T$

- who is subject to a suboptimal investment strategy generated from a CRRA utility function with risk aversion parameter $\bar{\gamma}$

is given by

$$
\widehat{W}_{0}=W_{0} e^{-\frac{1}{2 \gamma}\left(1-\frac{\gamma}{\gamma}\right)^{2} \eta^{2} T}
$$

The wealth equivalent $\widehat{W}_{0}$ for a long term investor with relative risk aversion parameter $\gamma$ significantly different from $\bar{\gamma}$ can be markedly less than $W_{0}$. It is clear from (16) that the magnitude of $\eta^{2} T$ is essential - the effect of choosing a wrong value of $\bar{\gamma}$ is quite sensitive to the length of the investment horizon as well as to the level of the risk premium $\eta$.

We show some numerical results in Table 1 for two investment horizons, $T=5$ years and $T=25$ years, respectively, and a risk premium $\eta=0.3$. I.e. $\eta^{2} T=0.45$ for $T=5$ and $\eta^{2} T=2.25$ for $T=25$. The value of $\eta$ is in line with what is normally assumed for this parameter. As is clear from Table 1, the degree of suboptimality increases as we move away from the diagonal and decreases as we move down in the southeast direction. The degree of suboptimality is modest for combinations like, e.g., $(\bar{\gamma}=5, \gamma=10)$, whereas an investment in accordance with the logarithmic investor's growth optimal portfolio policy is devastating for a CRRA investor with a markedly higher relative risk aversion parameter.

Insert Table 1 around here

3 The details of the proof have been worked out in Brennan and Torous (1999) and as Corollary 2 in Jensen and Sørensen (2001), as previously referred to. For completeness we derive this result independently in Appendix A. For an alternative approach using loss rates, see Balder and Mahayni (2010). 
The obvious way to find a compromise value $\bar{\gamma}$ in order to reach a "fair" solution - within the simple portfolio policies derived from the CRRA utility framework - is to require that the percentage reduction in the wealth equivalents are identical across the two investors. This leads to the geometric mean:

$$
\bar{\gamma}=\sqrt{\gamma_{1} \gamma_{2}}
$$

which by construction is independent of the initial wealth distribution. To illustrate the interplay between two CRRA investors in a common pool with this portfolio policy we show the effect of this choice of $\bar{\gamma}$ graphically in Figures 1 and 2 for the two cases of investment horizons.

Insert Figure 1 around here

Insert Figure 2 around here

We vary the individual relative risk aversion parameters on the two axes and show the common value of the wealth equivalent relative to the initial wealth level. The wealth equivalent in relative terms is independent of the initial distribution of wealth among the two investors. Since the relation is fully symmetric around the $\gamma_{1}=\gamma_{2}$ axis we only show that part of the graph where $\gamma_{1} \leq \gamma_{2}$. Along the "diagonal", where $\gamma_{1}=\gamma_{2}$, the level is one as the investors agree on the optimal policy. The grid used along both axes in both figures is a variation in $\gamma$ of 0.5 . The continuously drawn curves are interpolated between these points.

As shown in the graphs, the loss is rather small for small deviations in the risk aversion parameters $\left(\gamma_{1}, \gamma_{2}\right)$. Furthermore, the higher their degrees of relative risk aversion is, the less sensitive is the loss in wealth equivalent for these two investors. A few numerical examples: With $\gamma_{1}=4$ and $\gamma_{2}=7$ the wealth equivalent is $99.67 \%$ for the 5 year time horizon and $98.34 \%$ for the 25 year time horizon. And with $\gamma_{1}=2$ and $\gamma_{2}=8$ the wealth equivalent is $97.23 \%$ for the 5 year time horizon and $86.88 \%$ for the 25 year time horizon. If we pair $\gamma_{1}=3$ with $\gamma_{2}=10$ the wealth equivalents are $98.48 \%$ and $92.62 \%$, respectively. On the other hand, when the value of $\gamma_{1}$ is found in the range around the logarithmic investor $\left(\gamma_{1}=1\right)$ or even with a $\gamma_{1}$ value below unity, the effect can become quite outspoken. Coupling the logarithmic investor with $\gamma_{2}=10$ leads to the wealth equivalents $90.01 \%$ and $59.10 \%$, respectively. Coupling the logarithmic investor and the square root investor, i.e. $\left(\gamma_{1}=0.5, \gamma_{2}=1\right)$, which is a popular case in the literature on heterogeneous agents, leads to wealth equivalents of $96.21 \%$ and $82.45 \%$, respectively. The most extreme combination $\left(\gamma_{1}=0.5, \gamma_{2}=10\right)$ leads to $76.24 \%$ and $25.76 \%$, respectively.

A first order effect in this analysis is that when $\gamma$ reaches a certain level, all investors tend to have little exposure to the risky asset and tilt their portfolio towards the risk-free asset. Furthermore, their individually optimal portfolio composition varies much less with the value of $\gamma$ for relatively high levels of $\gamma$ than for relatively low levels of $\gamma$. This is due to the fact that the demand for risky assets varies proportional to the risk tolerance measure $1 / \gamma$. A first order effect in the opposite direction is that the investors have markedly different attitudes towards the extreme outcomes. In the low (high) wealth states, the Arrow-Debreu prices become very high (low) and the marginal utility for both investors tends to $+\infty$ (zero). However, the speed of convergence in marginal utilities is different for different investors; the higher the degree of risk aversion $\gamma$, the faster (slower) the convergence to $+\infty$ (zero) in the low (high) end of the wealth distribution.

\subsection{Constrained Pareto optimal portfolios}

When a linear sharing rule is given a priori, all constrained Pareto optimal policies are characterized by solving the following "central planner optimization problem", where $\mu \in[0,1]$ is a parameter that governs the relative importance of each of the investors in the pool:

$$
\begin{aligned}
& \max _{\mathcal{W}_{T}} E\left[\frac{\mu}{1-\gamma_{1}}\left(\phi \mathcal{W}_{T}\right)^{1-\gamma_{1}}+\frac{1-\mu}{1-\gamma_{2}}\left((1-\phi) \mathcal{W}_{T}\right)^{1-\gamma_{2}}\right] \\
& \text { s.t. } \\
& E\left[M_{T} \mathcal{W}_{T}\right]=W_{1,0}+W_{2,0} . \quad[\lambda]
\end{aligned}
$$


The solution to the central planner's optimization problem is a straightforward application of the martingale method. The optimal distribution of wealth $\mathcal{W}_{T}$ at the horizon $T$ for a given $\mu$ is characterized in terms of the pricing kernel $M_{T}$ as the solution to the following equation:

$$
\mu \phi^{1-\gamma_{1}} \mathcal{W}_{T}^{-\gamma_{1}}+(1-\mu)(1-\phi)^{1-\gamma_{2}} \mathcal{W}_{T}^{-\gamma_{2}}=\lambda M_{T}
$$

where $\lambda$ is determined by the budget constraint:

$$
E\left[M_{T} \mathcal{W}_{T}\right]=W_{1,0}+W_{2,0}
$$

Remark 4 The optimality condition (18) is the requirement that

$$
\mu \phi U_{1}^{\prime}\left(\phi \mathcal{W}_{T}\right)+(1-\mu)(1-\phi) U_{2}^{\prime}\left((1-\phi) \mathcal{W}_{T}\right)=\lambda M_{T}
$$

This is similar to the usual condition for a Pareto optimum. The only difference is that the sharing rule is required to be linear, resulting in a constrained Pareto optimal solution.

Remark 5 In the case $\gamma_{2}=2 \gamma_{1}$, Equation (18) is a second order polynomial in $\mathcal{W}_{T}^{-\gamma_{1}}$, cf. also the comments made in section 2 and Equation (15) in remark 3:

$$
\left[(1-\mu)(1-\phi)^{1-2 \gamma_{1}}\right]\left(\mathcal{W}_{T}^{-\gamma_{1}}\right)^{2}+\left[\mu \phi^{1-\gamma_{1}}\right] \mathcal{W}_{T}^{-\gamma_{1}}-\lambda M_{T}=0
$$

Using the standard formula for the roots of a second-order polynomial, the relevant solution can be written in closed form as:

$$
\begin{aligned}
\mathcal{W}_{T}^{-\gamma_{1}} & =\frac{-\mu \phi^{1-\gamma_{1}}+\sqrt{D}}{2(1-\mu)(1-\phi)^{1-2 \gamma_{1}}} \Leftrightarrow \mathcal{W}_{T}=\left[\frac{2(1-\mu)(1-\phi)^{1-2 \gamma_{1}}}{\sqrt{D}-\mu \phi^{1-\gamma_{1}}}\right]^{1 / \gamma_{1}} \\
D & =\mu^{2} \phi^{2\left(1-\gamma_{1}\right)}+4(1-\mu)(1-\phi)^{1-2 \gamma_{1}} \lambda M_{T} .
\end{aligned}
$$

This is markedly different from the standard model in the literature on heterogeneous agents in two respects. First, in an exchange economy context the aggregate output is given, but agents are allowed to enter into non-linear contracts in order to reach the unconstrained Pareto optimum. In our model, only linear contracts are allowed. Second, in our model the aggregate output for the two investors is not given a priori; rather, it is expressed in terms of the pricing kernel $M_{T}$ as a result of a constrained optimization problem.

Insert Figure 3 around here

Figure 3 shows the relation between two investors with markedly different levels of relative risk aversion, $\gamma_{1}=2$ and $\gamma_{2}=10$, respectively. The parameter values are $r=5 \%, \eta=0.3, T=5$. The values on the $x$-axis represent the aggregate wealth. The values on the $y$-axis represent the individual wealth levels in both the unconstrained scenario, where the two investors have been able to choose their portfolio freely, and in the constrained Pareto optimal scenario.

The investors have identical initial wealth $W_{0}=1$, i.e. $\phi=0.5$. The curves " $\gamma=2$ " and " $\gamma=10$ " show the constrained Pareto optimal portfolio choices for these two investors. In line with well known results from the literature on heterogeneous agents, the unconstrained sharing rule is a convex (concave) function of aggregate wealth for the investor with the lowest (highest) degree of relative risk aversion.

An example of the results from enforcing a linear sharing rule, the constrained Pareto optimal portfolio allocations, are shown in the two remaining curves. They show the result from arbitrarily setting the weighting parameter $\mu$ to 0.95 and 0.99 , respectively. ${ }^{4}$ These curves are not straight lines. This is because they are plotted against the unconstrained Pareto optimal solution on the first axis, illustrating the point that the optimal portfolio under constraints is different from the optimal portfolio resulting from the unconstrained market solution.

The curves show a general feature of this constrained Pareto optimal investment policy. In "bad states of the world" the investment policy is dominated by the investor with the highest degree of risk aversion, whereas in "good states of the world" it is dominated, or at least shaped, by the investor with the the lowest degree of risk aversion. Furthermore, the absolute deviation from each of the individual investors' first best investment portfolio increases. Similar results, however not shown, are obtained with a time horizon 25 years.

\footnotetext{
4 Note that the two utility functions behave rather differently, so an uncritical interpretation of the numerical value $\mu$ as
} a parameter that weighs the two investors against each other is not possible. 


\subsection{Fair distribution}

We have solved numerically ${ }^{5}$ for the optimal constrained investment policy, given that $\mu$ is chosen in such a manner that the relative loss in wealth equivalent is the same for both investors. We consider this to be the relevant interpretation of the concept "fair distribution" in this context. Hence, a numerical solution involves finding jointly the correct values of $\mu$ and $\lambda$ as functions of $\left(\gamma_{1}, \gamma_{2}\right)$ to fulfill this requirement.

Insert Table 2 around here

We consider two investors with identical initial wealth equal to one and show the results in terms of differences in their wealth equivalents in Table 2 with three decimals precision. Due to the symmetry, Table 2 only shows half the possible combinations and only for values of $\gamma_{2} \leq 3$; beyond that all entries are zeros up to three decimals precision. The solution should be better than the ad hoc rule analyzed in subsection 5.1. This is also the case. The differences are outspoken where the values of the risk aversion parameters paired consist of an investor with a risk aversion parameter below 1 and an investor with a relatively high risk aversion parameter. But the differences are very small and negligible, whenever both investors have risk aversion parameters exceeding, say, two. There is very little room for improvement as already seen in Figures 1 and 2; such combinations of investors tend to have a very similar perception of what the optimal unconstrained investment strategy should be, and the linear sharing rule is a minor restriction.

We illustrate the distribution of final wealth in Figures 4 through 6 . As part of our numerical procedure we have chosen a discretization of the sample space ${ }^{6}$ and, hence, the pricing kernel into 10,000 intervals of equal probability $10^{-4}$. The very last of these intervals is the right tail of the distribution cut off at the point where the tail probability is $10^{-4}$. The $x$-axis in Figures 4 through 6 corresponds to this discretisation, where the pricing kernel takes on the highest (lowest) values in the left (right) end of the graphs. Hence, the values on the $x$-axis are ordered in terms of fractiles, whereas the corresponding outcomes of the wealth process are represented on the $y$-axis.

The actual dynamic portfolio policy that implements the constrained Pareto optimal final wealth distribution is in no way an easy one to determine. It is not a policy with constant portfolio weights or a similar analytically convenient one. It can only be found by repeated intensive numerical calculations along the realisation with short time intervals. We have therefore chosen to illustrate our numerical results with probability steps on the $x$-axis to give a graphical view of the probabilities for small and large deviations, respectively. The closest we can get to an analytically convenient portfolio policy is the geometric mean policy with $\bar{\gamma}$ as described in subsection 5.1 and in Equation (17), for which the optimal portfolio policy has constant portfolio weights.

The dotted lines show the constrained Pareto optimal investment result for the two investors trading in a perfectly competitive financial market. The full drawn line is the result from using our fair constrained Pareto optimality criterion when linear sharing is required. It is clear from these figures that the deviations in the investment results are found primarily in the tails of the distribution.

A horizontal line in this representation indicates a portfolio policy with $100 \%$ investment in the risk-free asset. The more markedly S-shaped the curve is, the less risk averse the investor is. The figures show that the relatively least risk averse investor "bet on the right tail" and behave "put option like" in the left tail of the distribution of aggregate wealth, cf. also Figure 3 .

The most extreme combination we present is $\gamma_{1}=0.5, \gamma_{2}=10$. This combination is shown in Figure 4. Investor 2, the most risk averse, has a total variation in his consumption pattern going from 2.08 to 3.68 , whereas investor 1 has a total variation going from 0.02 to 1508.73. In order not to compress Figure 4 as a result of this extraordinary high level at the tail for investor 1 , we have cut of the graph at a level of approximately 800 .

Insert Figure 4 around here

\footnotetext{
5 There is no analytical solution available for this problem, even if the parameters are chosen in such a manner that the true Pareto optimal solution is available in analytical form. Hence, we are forced to rely on a numerical solution technique.

6 We tested the approximation on problems with known analytical solutions to check the accuracy. We found that the numerical solution and the analytical solution coincided with 4 decimals precision.
} 
The enforcement of the linear sharing rule clearly affects the level of wealth equivalents differently depending on the pairing of the values for their respective risk aversion parameters. The deviances between the two investors become less and less outspoken when the absolute level of risk aversion increases for both investors, despite that the individual values may still be significantly different. This is due to the fact that both investors have an increasing desire for an even wealth distribution, i.e., a preference for a large share of the riskless asset in the portfolio. In other words, when investor 1 has a high level of risk aversion, and investor 2 an even higher level, the loss due to the linear sharing rule is very limited; and a high level in this context is a value of $\gamma_{1}$, say, at or above 5. In Figure 5, this situation is presented for the combination $\left(\gamma_{1}, \gamma_{2}\right)=(5,10)$.

Insert Figure 5 around here

In the other end we see that if investor 1 has a value of, say, $\gamma_{1}=1$, the deviances can be significant, even for investor 2 with, say, $\gamma_{2}=2$. The difference is substantial for rather small absolute differences in the $\gamma$ values in this range. The combination $\left(\gamma_{1}, \gamma_{2}\right)=(0.5,1)$ was used in Dumas' seminal paper (Dumas $\left.(1989)\right)$ and has subsequently been repeated by others. Investors with such characteristics are suffering significant losses in their wealth equivalents by being forced into a common pool with others and to share the final result linearly. Mixing an investor 1 with a risk aversion parameter in the range around 1 with an investor 2 with a risk aversion parameter in the range 5 or more can, indeed, lead to severe losses in wealth equivalents.

In Figure 6 this situation is presented for the combination $\left(\gamma_{1}, \gamma_{2}\right)=(1,5)$.

Insert Figure 6 around here

In all the Figures 4 through 6 the deviations are clearly concentrated in the upper and lower tails of the distribution. In the middle of the probability distribution, the investors behave in rather similar manners and in such a way that their first best optimal distribution of final wealth is close the fair solution. We show in Table 3 the probability mass over which the first best solution deviate by more than $1 \%, 5 \%$ and $10 \%$, respectively, from the fair distribution. Furthermore, larger deviations in absolute terms are primarily concentrated in the right end of the graphs where Arrow-Debreu prices as well as marginal utilities are low. In the left end of the graphs, where the Arow-Debreu prices as well as the marginal utilities are high, the absolute deviations are much smaller.

Please insert Table 3 around here.

Hence, the restriction of a linear sharing rule does not affect the resulting asset allocation very much in this area. And as shown, the simple geometric mean rule is, in turn, close to this compromise solution in terms of wealth equivalents.

\subsection{Sensitivity analysis}

The optimal solution depends on the weighting of the preferences through the weighting parameter $\mu$. The results shown in Figure 7 assume that $\gamma_{2}>\gamma_{1}$, i.e. investor 2 is the relatively most risk averse investor. When the weight $\mu$ attached to investor 1 , the least risk averse investor, is increased the chosen asset allocation will decrease the outcomes in the "bad states", whereas the asset allocation in the "good states" will increase. The details are lengthy and, hence, we devote the derivations to Appendix B. Figure 7 illustrates this basic insight by showing that the elasticity of aggregate wealth, $\mathcal{W}$, with respect to the weighting parameter $\mu$ is negative in "bad states" and positive in "good states" with an asymptotic limit equal to $\frac{\frac{1}{1-\mu}-k}{\gamma_{1}}$. This asymptotic limit is increasing in $\mu$ and decreasing in $\gamma_{1}$.

Insert Figure 7 around here

This is in accordance with the existing literature on heterogeneous agent models, except for the fact that the asset allocation and the aggregate risk exposure is chosen by the investors in accordance with the stated joint objective function instead of being given as in an exchange economy context.

Deviating from the fair constrained Pareto optimal solution, it is tempting to suggest that the fund manager should choose a logarithmic utility function as he then will maximize the expected rate of return over any time horizon. However, the results we have presented show that such a policy may be severely wrong and that a fund manager with such a strategy can inflict serious utility losses on at least some investors. 


\section{Concluding remarks}

In this paper we have analyzed selection criteria for the situation where two investors are forced to invest in a common portfolio and share the proceeds by a linear sharing rule. The investment strategy is chosen by a manager in order to compromise between the two investors' preferences. This imposes two restrictions. One is the suboptimal investment strategy as seen from each of the investors' point of view. The other is the required linear sharing rule. A similar situation with many investors is typical for defined contribution pension schemes.

Both investors were assumed to possess a CRRA utility function; this choice of preferences made it possible to use analytically known solutions as a benchmark to measure the loss in wealth equivalent.

We analyzed two settings. In one setting the manager was supposed to make investment decisions in accordance with a CRRA utility function, where the risk aversion parameter was the geometric mean of the two individual investors' risk aversion parameters. This choice implied an easily implementable portfolio choice policy, namely a constant share of wealth allocated to the risky asset, and it guarantees that the relative loss in wealth equivalent is the same for both investors. We observe large losses in wealth equivalents if the risk aversion parameters were very different and one of them quite small, say logarithmic $(\gamma=1)$ or less. On the other hand, when both investors have relatively high risk aversion parameters the loss in their wealth equivalents is hardly observable.

In the other setting we searched numerically for the constrained Pareto optimal solution which produced the same relative loss in wealth equivalent for each of the two investors. Compared to the former setting some gains were observed, but - again - only in cases where the risk aversion parameters were very different and one of them quite small.

One observation is worth mentioning. It is sometimes argued that a manager behaving like the logarithmic investor $(\gamma=1)$ is a good choice for such a common investment problem, because he focuses on the expected long term rate of return. Our results show that such a manager can impose severe losses on the individual investors whenever they have a risk attitude more appropriately described by a relative risk aversion parameter somewhat higher, say $\gamma>3$. There are also severe losses for investors on the other side like, e.g., the square root type with $\gamma=0.5$. We have observed that the individual optimal behavior of investors with such low degrees of risk aversion is almost literally like the "tail wagging the dog".

\section{References}

Balder, S., Mahayni, A., 2010. How good is portfolio insurance? In: Kiesel, R., Scherer, M., Zagst, R. (Eds.), Alternative Investments and Strategies. World Scientific, pp. 229-256.

Benninga, S., Mayshar, J., 2000. Heterogeneity and option pricing. Review of Derivatives Research 4, 7-27.

Brennan, M. J., Torous, W. N., 1999. Individual decision making and investor welfare. Economic Notes by Banca Monte dei Paschi di Siena SpA, 119-143.

Cox, J. C., Ingersoll, Jr., J. E., Ross, S. A., Mar. 1985. An intertemporal general equilibrium model of asset prices. Econometrica 53 (2), 363-384.

Cvitanić, J., Jouini, E., Malamud, S., Napp, C., 2012. Financial markets equilibrium with heterogenous agents. Review of Finance 16 (1), 285-321.

Duffie, D., 1996. Dynamic Asset Pricing Theory. Princeton University Press, Princeton, New Jersey, USA, 2nd edition.

Dumas, B., 1989. Two-person dynamic equilibrium in the capital market. The Review of Financial Studies 2, $157-188$.

Flor, C. R., Larsen, L. S., 2014. Robust portfolio choice with stochastic interest rates. Annals of Finance 10, 243-265.

Franke, G., Lüders, E., 2010. Return predictibality and stock market crashes in a simple rational expectations model. Advances in Decision Sciences 2010, Article ID 791025, 27 pages, doi:10.1155/2010/791025.

Franke, G., Stapleton, R. C., Subrahmanyam, M. G., 1998. Who buys and who sells options: The role of options in an economy with background risk. Journal of Economic Theory 82, 89-109.

Franke, G., Stapleton, R. C., Subrahmanyam, M. G., 1999. When are options overpriced? The Black-Scholes model and alternative characterisations of the pricing kernel. European Finance Review 3, 79-102.

Hara, C., 2006. Heterogeneous risk attitudes in a continuous-time model. Japanese Economic Review 57, $377-405$. 
Hara, C., Huang, J., Kuzmics, C., 2007. Representative consumer's risk aversion and efficient sharing rules. Journal of Economic Theory 137, 652-672.

Jensen, B. A., Sørensen, C., 2001. Paying for minimum interest rate guarantees: Who should compensate who? European Financial Management 7 (2), 183-211.

Larsen, L. S., Munk, C., 2012. The costs of suboptimal dynamic asset allocation: General rsults and applications to interest rate risk, stock volatility, and growth/value tilts. Journal of Economic Dynamics and Control 36, 266-293.

Merton, R. C., Dec. 1971. Optimum consumption and portfolio rules in a continuous time model. Journal of Economic Theory 3, 373-413, erratum: Merton (1973). Reprinted in (Merton, 1992, Chapter 5).

Merton, R. C., 1973. Erratum. Journal of Economic Theory 6, 213-214.

Merton, R. C., 1992. Continuous-Time Finance. Basil Blackwell Inc., Cambridge, Massachusetts.

Rogers, L., 2001. The relaxed investor and parameter uncertainty. Finance and Stochastics 5, 131-154.

Stapleton, R. C., Subrahmanyam, M. G., 1990. Risk aversion and the intertemporal behavior of asset prices. The Review of Financial Studies 3, 677-693.

Wang, J., 1996. The term structure of interest rates in a pure exchange economy with heterogeneous investors. Journal of Financial Economics 41, 75-110.

Weinbaum, D., 2009. Investor heterogeneity, asset pricing and volatility dynamics. Journal of Economic Dynamics and Control 33, 1379-1397.

We would like to thank Marcel Fischer, Antje Mahayni and an anonymous referee for useful comments on earlier versions of the manuscript. We are grateful for financial support from the Pension Research Center (PerCent) at Copenhagen Business School. 


\section{Appendix}

\section{A Proof of Theorem 3}

For any given constant proportional portfolio policy with portfolio weight $\pi$ allocated to the risky asset, the terminal wealth is, cf. Equation (5):

$$
W_{T}=W_{0} e^{\left(r+\pi \eta \sigma-\frac{1}{2} \pi^{2} \sigma^{2}\right) T+\pi \sigma Z_{T}} .
$$

Routine calculations with expected values of log-normal distributions lead to:

$$
\begin{aligned}
W_{T}^{1-\gamma} & =W_{0}^{1-\gamma} e^{(1-\gamma)\left(r+\pi \eta \sigma-\frac{1}{2} \pi^{2} \sigma^{2}\right) T+(1-\gamma) \pi \sigma Z_{T}} \\
\mathbb{E}\left[W_{T}^{1-\gamma}\right] & =W_{0}^{1-\gamma} e^{(1-\gamma)\left(r+\pi \eta \sigma-\frac{1}{2} \pi^{2} \sigma^{2}+\frac{1}{2}(1-\gamma) \pi^{2} \sigma^{2}\right) T} \\
& =W_{0}^{1-\gamma} e^{(1-\gamma)\left(r+\pi \eta \sigma-\frac{\gamma}{2} \pi^{2} \sigma^{2}\right) T} .
\end{aligned}
$$

The optimal portfolio weight $\pi$ for any given value of $\gamma$ is found by maximizing the expression $\pi \eta \sigma-\frac{\gamma}{2} \pi^{2} \sigma^{2}$, which leads to the classical result that $\pi=\frac{\eta}{\gamma \sigma}$.

If a suboptimal portfolio weight is imposed, arising from an "erroneous" choice of $\bar{\gamma}$ instead of the "true" $\gamma$, we can insert $\bar{\pi}=\frac{\eta}{\bar{\gamma} \sigma}$ in Equation (A.4) and get the following closed form expression for the expected utility:

$$
\mathbb{E}\left[W_{T}^{1-\gamma}\right]=W_{0}^{1-\gamma} e^{(1-\gamma)\left(r+\frac{\eta^{2}}{\bar{\gamma}}-\frac{\gamma}{2} \frac{\eta^{2}}{\bar{\gamma}^{2}}\right) T} .
$$

Compare this with an optimally invested inital wealth $\widehat{W}_{0}$ :

$$
\mathbb{E}\left[\widehat{W}_{T}^{1-\gamma}\right]=\widehat{W}_{0}^{1-\gamma} e^{(1-\gamma)\left(r+\frac{\eta^{2}}{\gamma}-\frac{\gamma}{2} \frac{\eta^{2}}{\gamma^{2}}\right) T} .
$$

The wealth equivalent is the magnitude of $\widehat{W}_{0}$ that equates the expected utility in Equation (A.5) with the expected utility in Equation (A.6). That is:

$$
\frac{\widehat{W}_{0}}{W_{0}}=e^{\left(\frac{\eta^{2}}{\bar{\gamma}}-\frac{\gamma}{2} \frac{\eta^{2}}{\bar{\gamma}^{2}}\right) T-\left(\frac{\eta^{2}}{\gamma}-\frac{\gamma}{2} \frac{\eta^{2}}{\gamma^{2}}\right) T}=e^{\frac{-\eta^{2} T}{2 \gamma}\left(\frac{-2 \gamma}{\bar{\gamma}}+\frac{\gamma^{2}}{\bar{\gamma}^{2}}+1\right)}=e^{-\frac{1}{2 \gamma}\left(1-\frac{\gamma}{\bar{\gamma}}\right)^{2} \eta^{2} T}
$$

\section{B The derivations behind Figure 7}

The Pareto optimal allocation is clearly affected by the choice of $\mu$ as shown in Figure 3. The technical derivations behind Figure 7 are given here. The starting point is the first order condition (18), which is subjected to a series of differentiations. We denote the conglomerate utility function of the "representative investor" by $\mathcal{U}$.

$$
\begin{aligned}
\lambda M_{T}= & \mu \xi^{1-\gamma_{1}} \mathcal{W}^{-\gamma_{1}}+(1-\mu)(1-\xi)^{1-\gamma_{2}} \mathcal{W}^{-\gamma_{2}}\left(=\mathcal{U}^{\prime}(\mathcal{W})\right) \\
\frac{\partial \lambda}{\partial \mu} M_{T}= & {\left[\mu \xi^{1-\gamma_{1}}\left(-\gamma_{1}\right) \mathcal{W}^{-\gamma_{1}-1}+(1-\mu)(1-\xi)^{1-\gamma_{2}}\left(-\gamma_{2}\right) \mathcal{W}^{-\gamma_{2}-1}\right] \frac{\partial \mathcal{W}}{\partial \mu}+} \\
& \xi^{1-\gamma_{1}} \mathcal{W}^{-\gamma_{1}}-(1-\xi)^{1-\gamma_{2}} \mathcal{W}^{-\gamma_{2}} \quad\left(=\frac{\partial \mathcal{U}^{\prime}(\mathcal{W})}{\partial \mu}\right) \\
\lambda \frac{\partial M_{T}}{\partial \mathcal{W}}= & {\left[\mu \xi^{1-\gamma_{1}}\left(-\gamma_{1}\right) \mathcal{W}^{-\gamma_{1}-1}+(1-\mu)(1-\xi)^{1-\gamma_{2}}\left(-\gamma_{2}\right) \mathcal{W}^{-\gamma_{2}-1}\right] \quad\left(=\mathcal{U}^{\prime \prime}(\mathcal{W})\right) . }
\end{aligned}
$$

Upon dividing (A.10) by (A.8) we arrive at the following expression for the relative risk aversion of the "representative investor"

$$
-\frac{\partial M_{T}}{\partial \mathcal{W}} \frac{\mathcal{W}}{M_{T}}=-\frac{\mathcal{U}^{\prime \prime}(\mathcal{W}) \mathcal{W}}{\mathcal{U}^{\prime}(\mathcal{W})}=\Phi_{1} \gamma_{1}+\Phi_{2} \gamma_{2}(\equiv \mathcal{R}(\mathcal{W}))
$$

where

$$
\Phi_{1}=\frac{\mu \xi^{1-\gamma_{1}} \mathcal{W}^{-\gamma_{1}}}{\mu \xi^{1-\gamma_{1}} \mathcal{W}^{-\gamma_{1}}+(1-\mu)(1-\xi)^{1-\gamma_{2}} \mathcal{W}^{-\gamma_{2}}}, \quad \Phi_{2}=1-\Phi_{1}
$$

Relation (A.11) shows that the representative investor has a relative risk aversion $\mathcal{R}(\mathcal{W})$ equal to an arithmetically weighted average of the two $\gamma$-values with weights given by their contribution to the central planner's marginal utility of wealth. ${ }^{7}$

7 In some papers on heterogeneous agent models, see e.g. Benninga and Mayshar (2000), it is claimed that the RRA for the representative investor is a harmonic average of the two individual RRAs. In this representation the average is arithmetic. Of course, for any harmonic average there is an equivalent arithmetic average, just with other weights. 
Furthermore, by dividing (A.9) by (A.8) and making use of the relations in (A.12) defining the weights $\Phi_{1}$ and $\Phi_{2}=1-\Phi_{1}$ we get the following relation:

$$
\frac{\partial \lambda}{\partial \mu} \frac{1}{\lambda}=-\mathcal{R}(\mathcal{W}) \frac{\partial \mathcal{W}}{\partial \mu} \frac{1}{\mathcal{W}}+\frac{\Phi_{1}}{\mu}-\frac{\Phi_{2}}{1-\mu} \quad \Leftrightarrow \quad \frac{\partial \lambda}{\partial \mu} \frac{\mu}{\lambda}=-\mathcal{R}(\mathcal{W}) \frac{\partial \mathcal{W}}{\partial \mu} \frac{1}{\mathcal{W}}+\frac{\Phi_{1}}{1-\mu}-\frac{\mu}{1-\mu} .
$$

The variable $\lambda$ is the Lagrangian multiplier for the budget constraint; it depends on the weight $\mu$, but is clearly independent of any particular realization $\mathcal{W}$ :

$$
\mathcal{R}(\mathcal{W}) \frac{\partial \mathcal{W}}{\partial \mu} \frac{\mu}{\mathcal{W}}+\underbrace{\frac{\partial \lambda}{\partial \mu} \frac{\mu}{\lambda}+\frac{\mu}{1-\mu}}_{\text {constant across } \mathrm{W}} \equiv \frac{\Phi_{1}}{1-\mu} .
$$

Let $\Psi(\mathcal{W})$ denote the elasticity of $\mathcal{W}$ with respect to the weighting parameter $\mu$ :

$$
\Psi(\mathcal{W}) \equiv \frac{\partial \mathcal{W}}{\partial \mu} \frac{\mu}{\mathcal{W}}
$$

and define $k$ as:

$$
k=\frac{\partial \lambda}{\partial \mu} \frac{\mu}{\lambda}+\frac{\mu}{1-\mu}
$$

Then relation (A.14) turns into:

$$
\mathcal{R}(\mathcal{W}) \Psi(\mathcal{W})=\frac{\Phi_{1}}{1-\mu}-k
$$

Assume without loss of generality that $\gamma_{1}<\gamma_{2}$. Then it follows from (A.12) that $\Phi_{1} \nearrow 1$ as $\mathcal{W} \nearrow \infty$, i.e. the least risk averse individual dominates the right hand tail. In the other end $\Phi_{1} \searrow 0$ as $\mathcal{W} \searrow 0$.

It can be ruled out that $k$ can be negative. In that case $\Psi(\mathcal{W})$ must always be positive, which will violate the budget constraint. Hence we can conclude that

$$
\Psi(\mathcal{W}) \rightarrow \begin{cases}-\frac{k}{\gamma_{2}} & (<0 \text { for } \mathcal{W} \rightarrow 0) \\ \frac{1}{\frac{1-\mu}{\gamma_{1}}-k} & (>0 \text { for } \mathcal{W} \rightarrow \infty)\end{cases}
$$


1

2

3

4

5

6

7

8

9

10

11

12

13

14

15

16

17

18

19

20

21

22

23

24

25

26

27

28

29

30

31

32

33

34

35

36

37

38

39

40

41

42

43

44

45

46

47

48

49

50

51

52

53

54

55

56

57

58

59

60

61

62

63

64

65

\begin{tabular}{|c|ccccc||ccccc|}
\hline & \multicolumn{7}{|c||}{$T=5$} & \multicolumn{5}{|c}{$T=25$} \\
$\gamma \backslash \bar{\gamma}$ & 0.5 & 1 & 2 & 5 & 10 & 0.5 & 1 & 2 & 5 & 10 \\
\hline 0.5 & 1 & 0.894 & 0.776 & 0.695 & 0.666 & 1 & 0.570 & 0.282 & 0.162 & 0.131 \\
1 & 0.799 & 1 & 0.945 & 0.866 & 0.833 & 0.325 & 1 & 0.755 & 0.487 & 0.402 \\
2 & 0.363 & 0.894 & 1 & 0.960 & 0.931 & 0.006 & 0.570 & 1 & 0.817 & 0.698 \\
5 & 0.026 & 0.487 & 0.904 & 1 & 0.989 & 0.000 & 0.027 & 0.603 & 1 & 0.945 \\
10 & 0.000 & 0.161 & 0.697 & 0.978 & 1 & 0.000 & 0.000 & 0.165 & 0.894 & 1 \\
\hline
\end{tabular}

Table 1 Wealth equivalents for investors with CRRA preferences $(\gamma)$ subject to an investment policy based on a wrong relative risk aversion $(\bar{\gamma})$ 
1

2

3

4

5

6

7

8

9

10

11

12

13

14

15

16

17

18

19

20

21

22

23

24

25

26

27

28

29

30

31

32

33

34

35

36

37

38

39

40

41

42

43

44

45

46

47

48

49

50

51

52

53

54

55

56

57

58

59

60

61

62

63

64

65

\begin{tabular}{|ccccccc|}
\hline$\gamma_{1} / \gamma_{2}$ & 0.5 & 1 & 1.5 & 2 & 2.5 & 3 \\
\hline 1.0 & 0.001 & & & & & \\
1.5 & 0.005 & 0 & & & & \\
2.0 & 0.011 & 0.001 & 0 & & & \\
2.5 & 0.016 & 0.001 & 0 & 0 & & \\
3.0 & 0.022 & 0.003 & 0 & 0 & 0 & \\
4.0 & 0.032 & 0.005 & 0.001 & 0 & 0 & 0 \\
5.0 & 0.041 & 0.008 & 0.002 & 0.001 & 0 & 0 \\
6.0 & 0.049 & 0.011 & 0.004 & 0.001 & 0.001 & 0 \\
7.0 & 0.055 & 0.014 & 0.005 & 0.002 & 0.001 & 0 \\
8.0 & 0.060 & 0.016 & 0.006 & 0.003 & 0.001 & 0.001 \\
9.0 & 0.065 & 0.019 & 0.007 & 0.003 & 0.002 & 0.001 \\
10 & 0.069 & 0.021 & 0.009 & 0.004 & 0.002 & 0.001 \\
\hline
\end{tabular}

Table 2 Differences in wealth equivalents for investors with CRRA preferences $\left(\gamma_{1}, \gamma_{2}\right)$ between the constrained Pareto optimal investment policy and an investment policy based on the square root rule $\left(\bar{\gamma}=\sqrt{\gamma_{1} \gamma_{2}}\right)$. 
1

\begin{tabular}{|c|ccc|}
\hline & $10 \%$ & $5 \%$ & $1 \%$ \\
\hline$\gamma_{1}=0.5, \gamma_{2}=10$ & & & \\
$p_{\gamma_{1}}$ & $6.99 \%$ & $3.49 \%$ & $0.69 \%$ \\
$p_{\gamma_{2}}$ & $15.53 \%$ & $7.11 \%$ & $1.40 \%$ \\
\hline$\gamma_{1}=1, \gamma_{2}=5$ & & & \\
$p_{\gamma_{l}}$ & $18.73 \%$ & $9.40 \%$ & $1.90 \%$ \\
$p_{\gamma_{2}}$ & $43.96 \%$ & $22.26 \%$ & $4.47 \%$ \\
\hline$\gamma_{1}=5, \gamma_{2}=10$ & & & \\
$p_{\gamma_{l}}$ & $99.75 \%$ & $74.24 \%$ & $16.04 \%$ \\
$p_{\gamma_{2}}$ & $98.65 \%$ & $84.65 \%$ & $24.89 \%$ \\
\hline
\end{tabular}

Table 3 Probabilities for being less than 1\%, 5\% and 10\%, respectively, away from the theoretically optimal distribution of terminal wealth. The entry marked with italics only take place in the area where the pricing kernel has low values, i.e. to the right in Figure 5. 


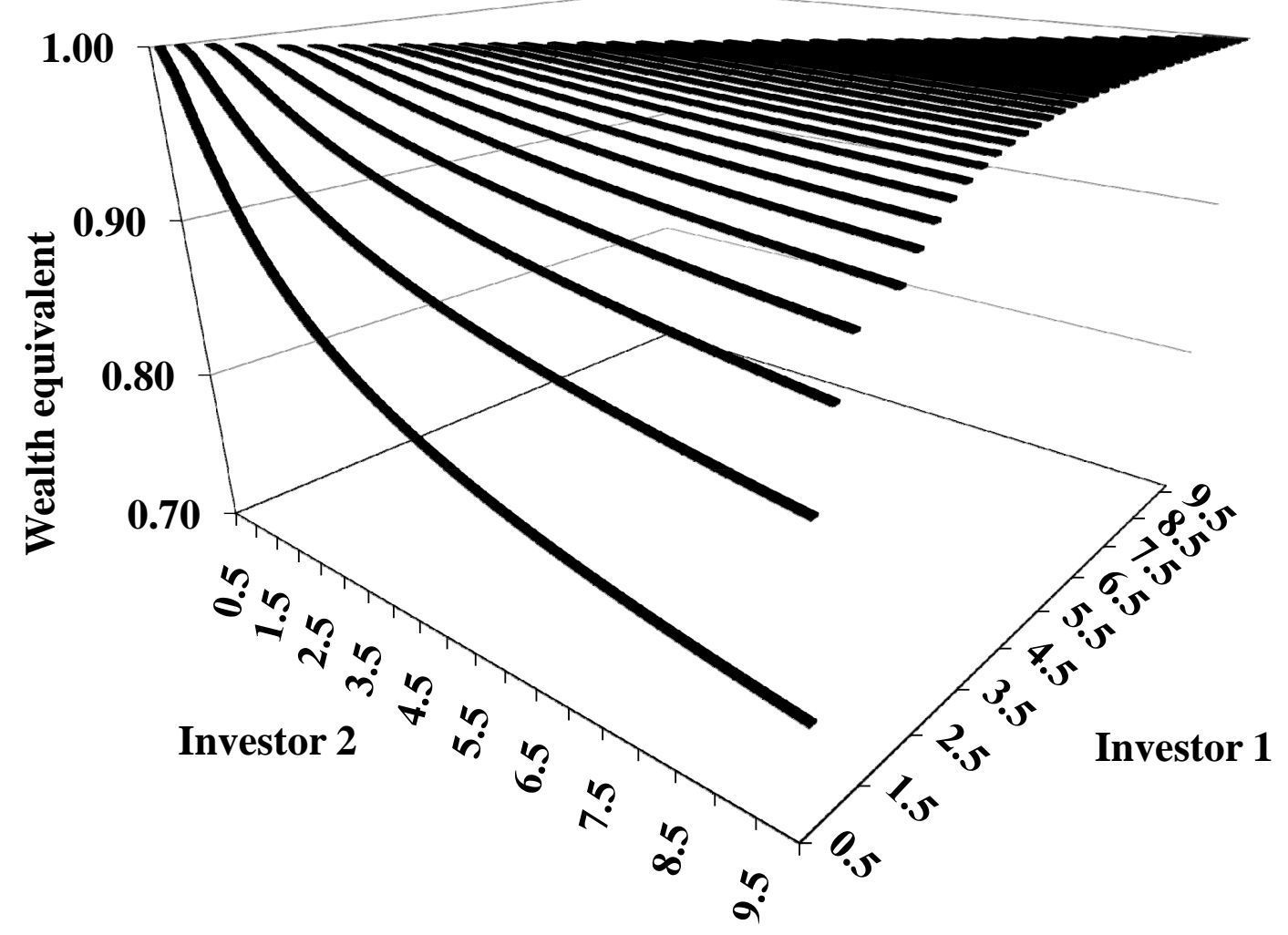

Figure 1 Wealth equivalent for investors participating in an investment pool with portfolio policies determined by equal relative reduction. Time horizon 5 years. 


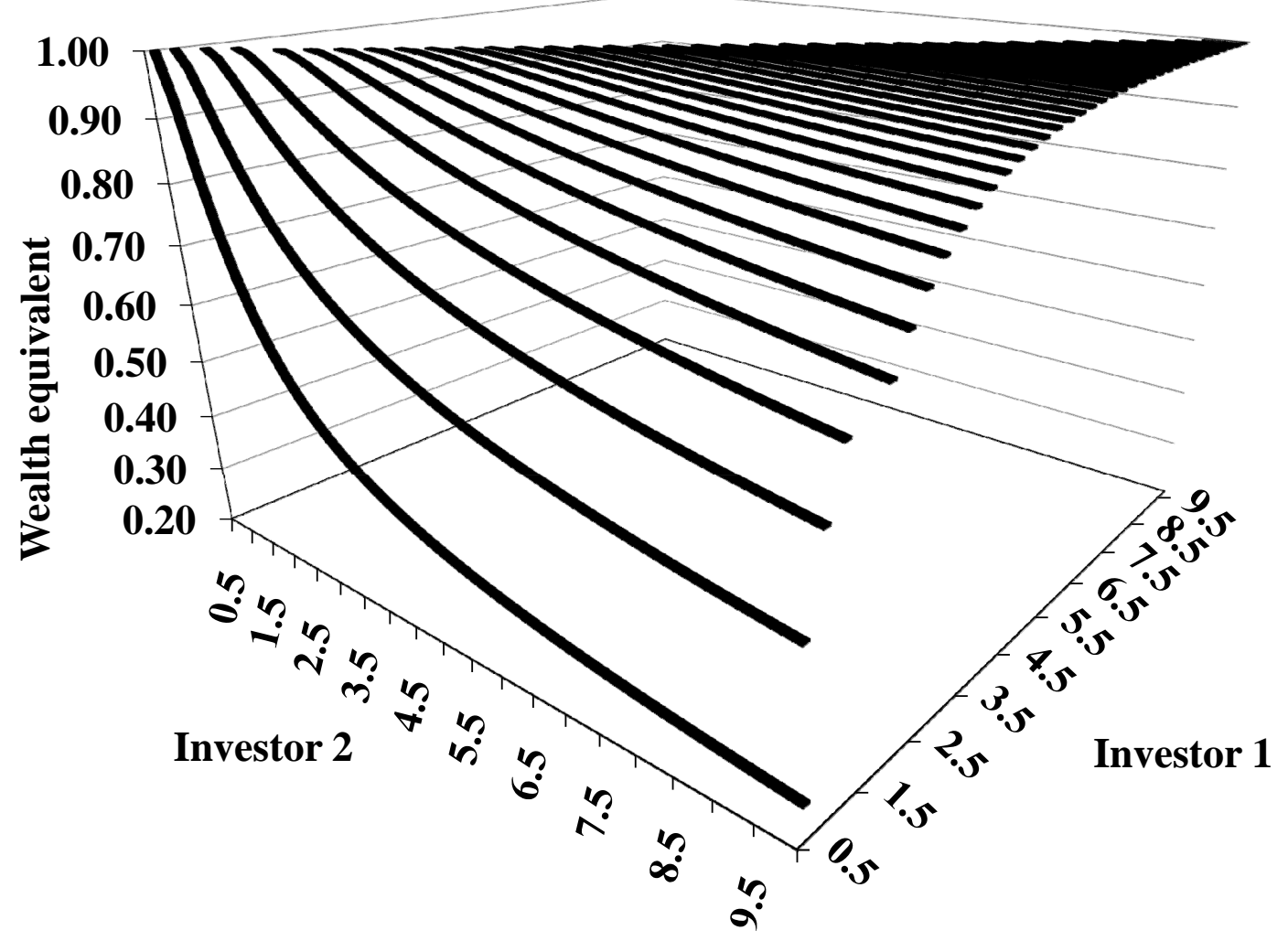

Figure 2 Wealth equivalent for investors participating in an investment pool with portfolio policies determined by equal relative reduction. Time horizon 25 years. 
1

2

3

4

5

6

7

8

9

10

11

12

13

14

15

16

17

18

19

20

21

22

23

24

25

26

27

28

29

30

31

32

33

34

35

36

37

38

39

40

41

42

43

44

45

46

47

48

49

50

51

52

53

54

55

56

57

58

59

60

61

62

63

64

65

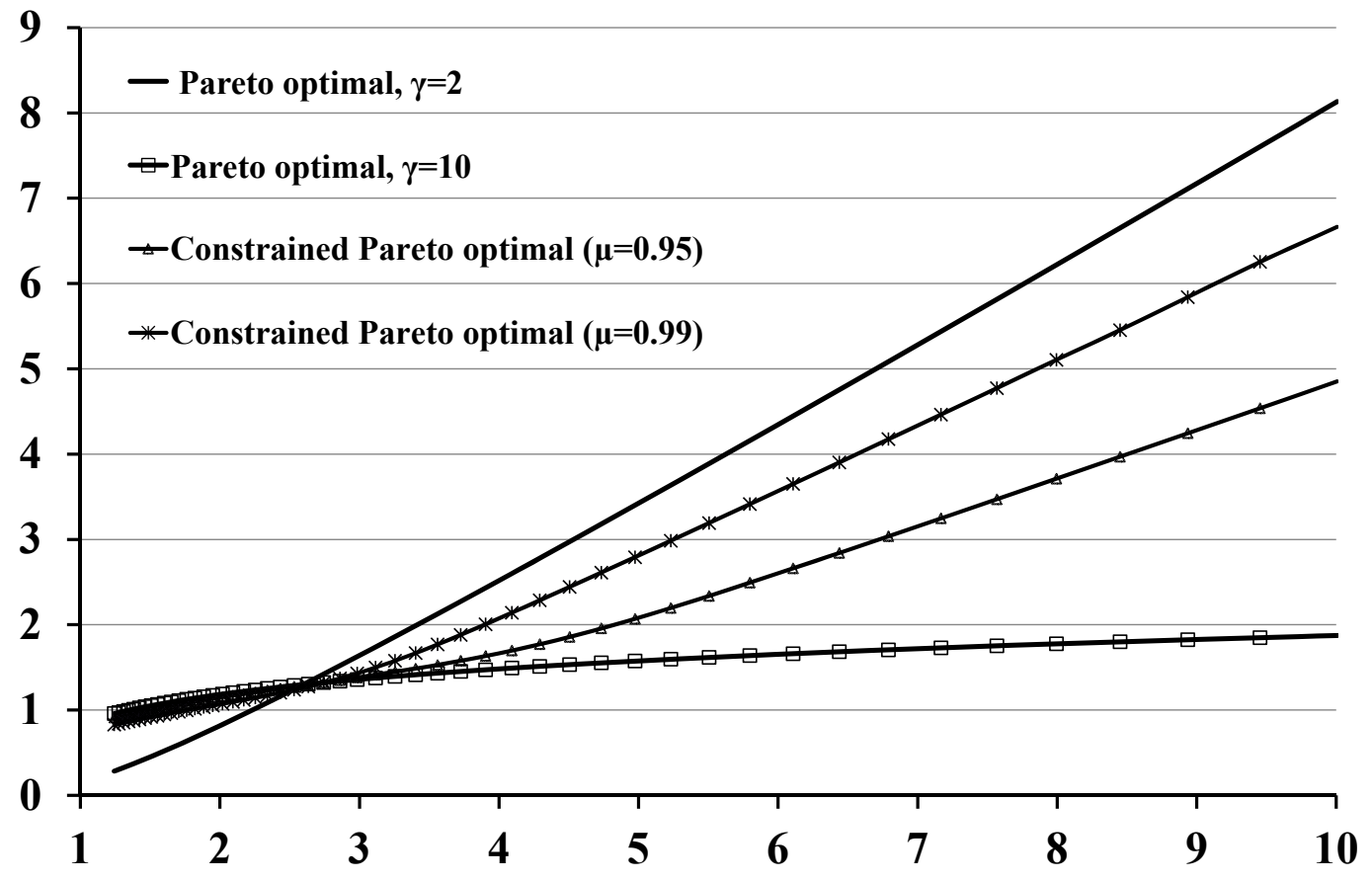

Figure 3 The $x$-axis shows aggregate wealth for investors participating in an investment pool. The $y$-axis shows the individual wealth levels for both the unconstrained scenario and the constrained Pareto optimal scenario determined by different values of the weighting parameter $\mu$. Time horizon 5 years. 
1

2

3

4

5

6

7

9

10

11

12

13

14

15

16

17

18

19

20

21

22

23

24

25

26

27

28

29

30

31

32

33

34

35

36

37

38

39

40

41

42

43

44

45

46

47

48

49

50

51

52

53

54

55

56

57

58

59

60

61

62

63

64

65

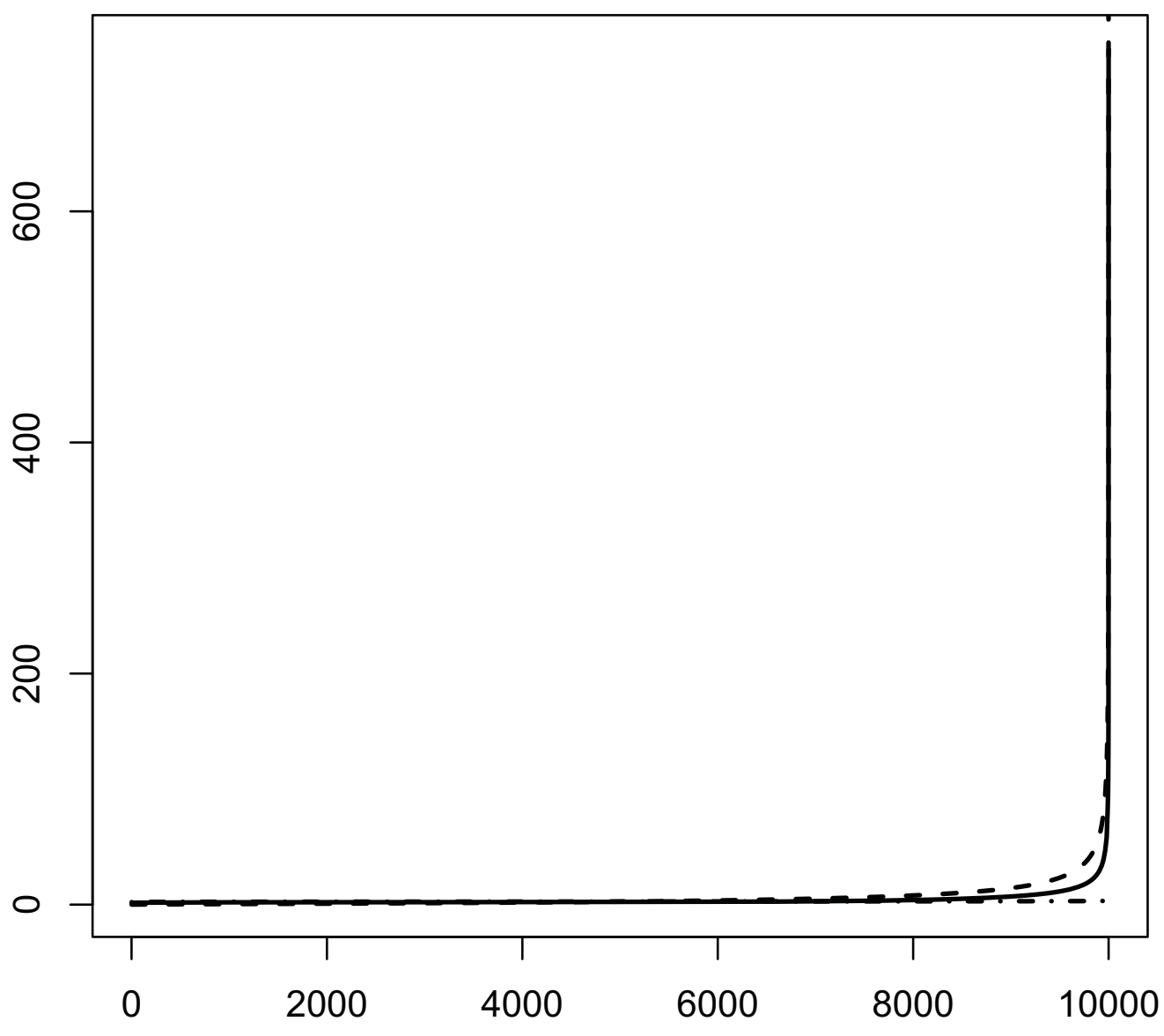

Figure 4 Allocation patterns for investors with $\left(\gamma_{1}, \gamma_{2}\right)=(0.5,10)$. The $x$-axis is divided into equal probability steps. The dotted lines show the unconstrained Pareto optimal final wealth for the two investors, whereas the solid curve shows the constrained Pareto optimal solution. 
1

2

3

4

5

6

7

8

9

10

11

12

13

14

15

16

17

18

19

20

21

22

23

24

25

26

27

28

29

30

31

32

33

34

35

36

37

38

39

40

41

42

43

44

45

46

47

48

49

50

51

52

53

54

55

56

57

58

59

60

61

62

63

64

65

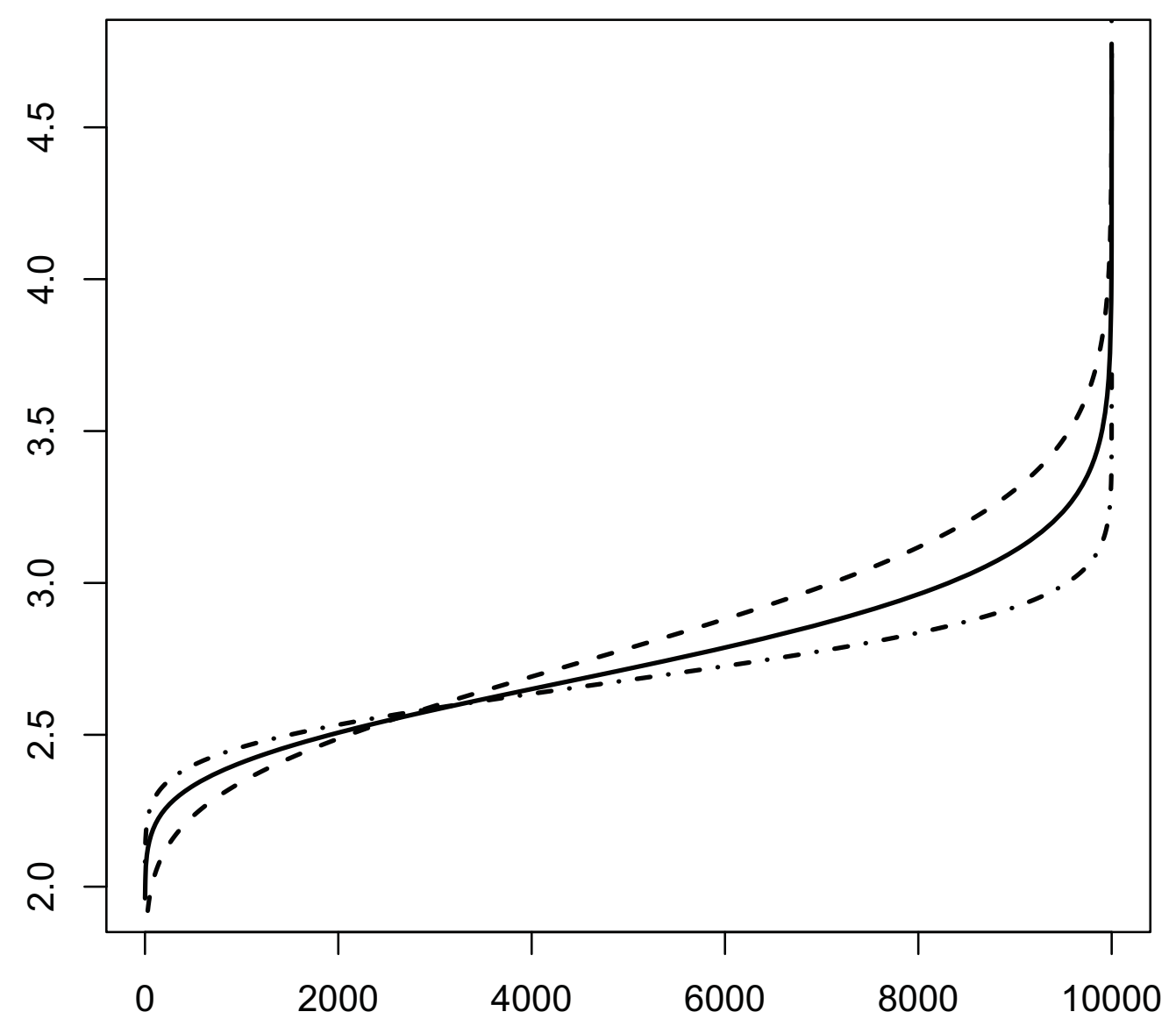

Figure 5 Allocation patterns for investors with $\left(\gamma_{1}, \gamma_{2}\right)=(5,10)$. The $x$-axis is divided into equal probability steps. The dotted lines show the unconstrained Pareto optimal final wealth for the two investors, whereas the solid curve shows the constrained Pareto optimal solution. 
1

2

3

4

5

6

7

8

9

10

11

12

13

14

15

16

17

18

19

20

21

22

23

24

25

26

27

28

29

30

31

32

33

34

35

36

37

38

39

40

41

42

43

44

45

46

47

48

49

50

51

52

53

54

55

56

57

58

59

60

61

62

63

64

65

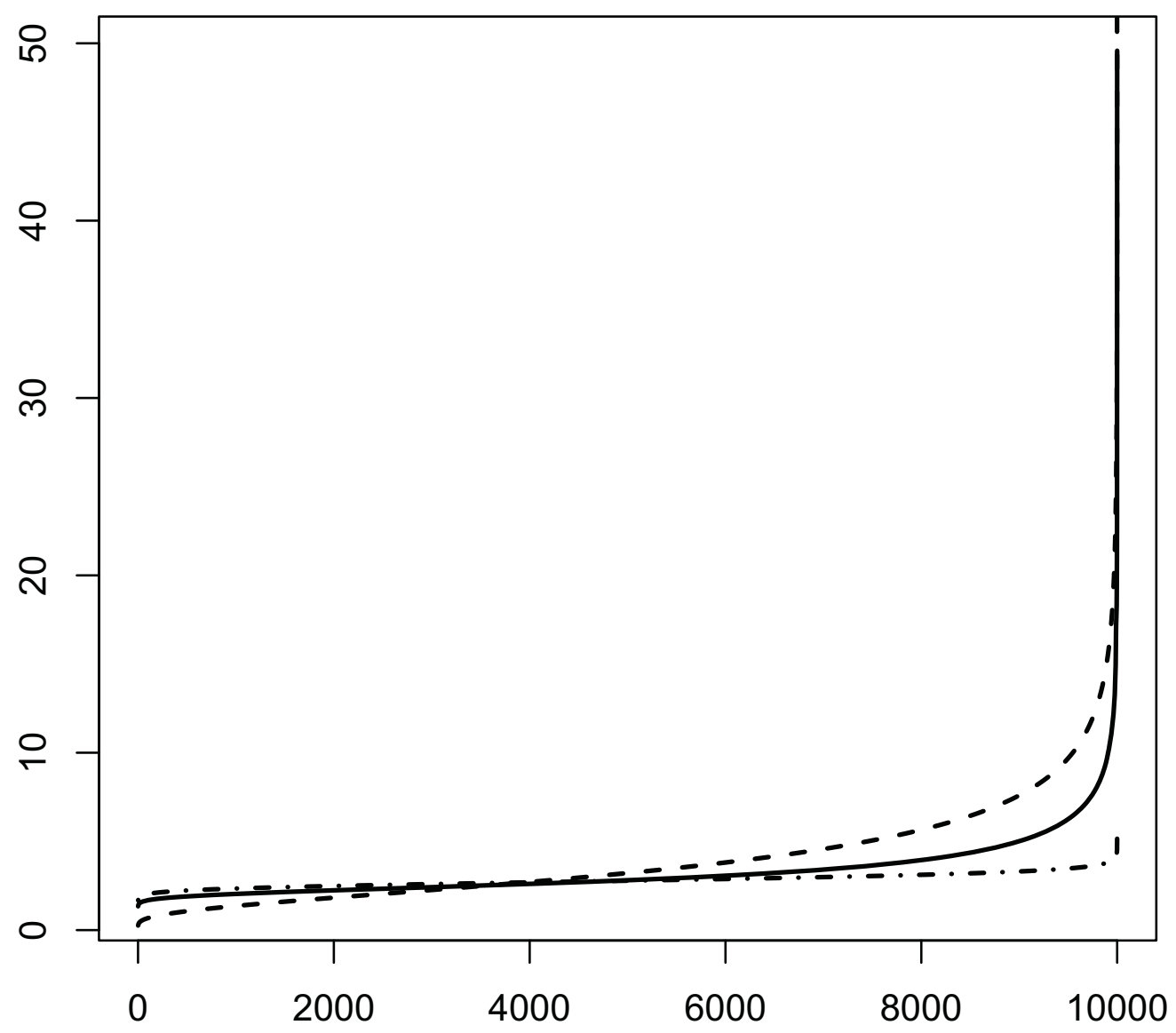

Figure 6 Allocation patterns for investors with $\left(\gamma_{1}, \gamma_{2}\right)=(1,5)$. The $x$-axis is divided into equal probability steps. The dotted lines show the unconstrained Pareto optimal final wealth for the two investors, whereas the solid curve shows the constrained Pareto optimal solution. 
1

2

3

4

5

6

7

8

9

10

11

12

13

14

15

16

17

18

19

20

21

22

23

24

25

26

27

28

29

30

31

32

33

34

35

36

37

38

39

40

41

42

43

44

45

46

47

48

49

50

51

52

53

54

55

56

57

58

59

60

61

62

63

64

65

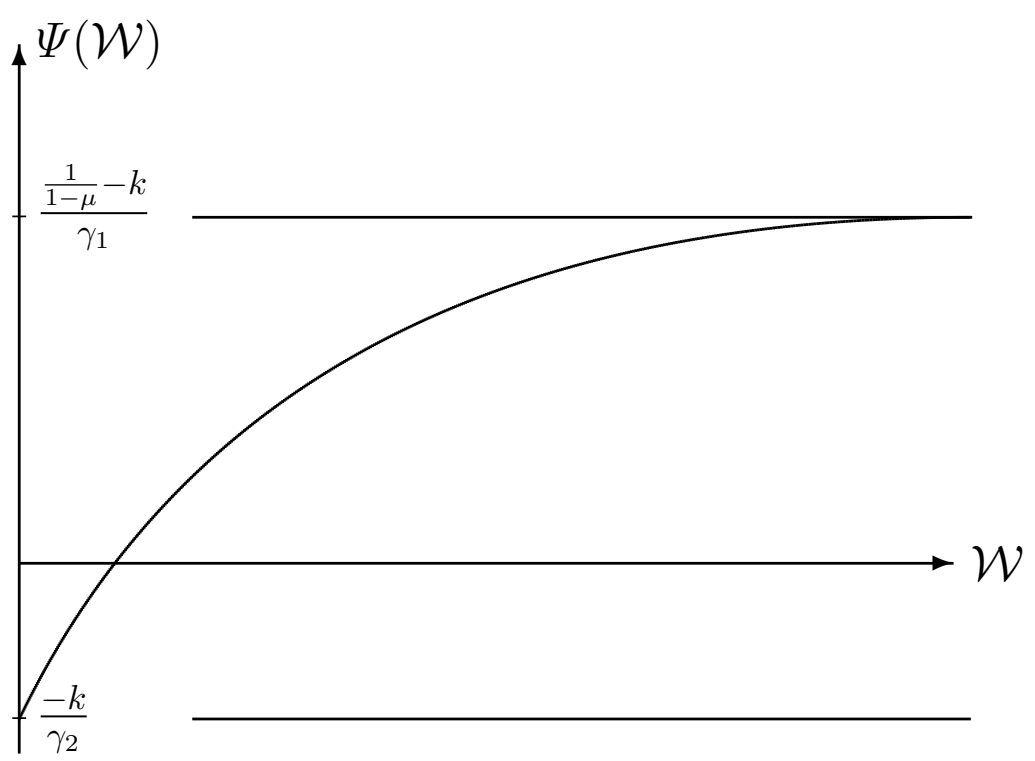

Figure 7 Sensitivity analysis: Variation in the elasticity $\Psi(\mathcal{W})$ of $\mathcal{W}$ with respect to the weighting parameter $\mu$ as a function of aggregate wealth. 
Click here to download Figure fig1.pdf $\underline{\underline{ }}$ Wealth equivalent

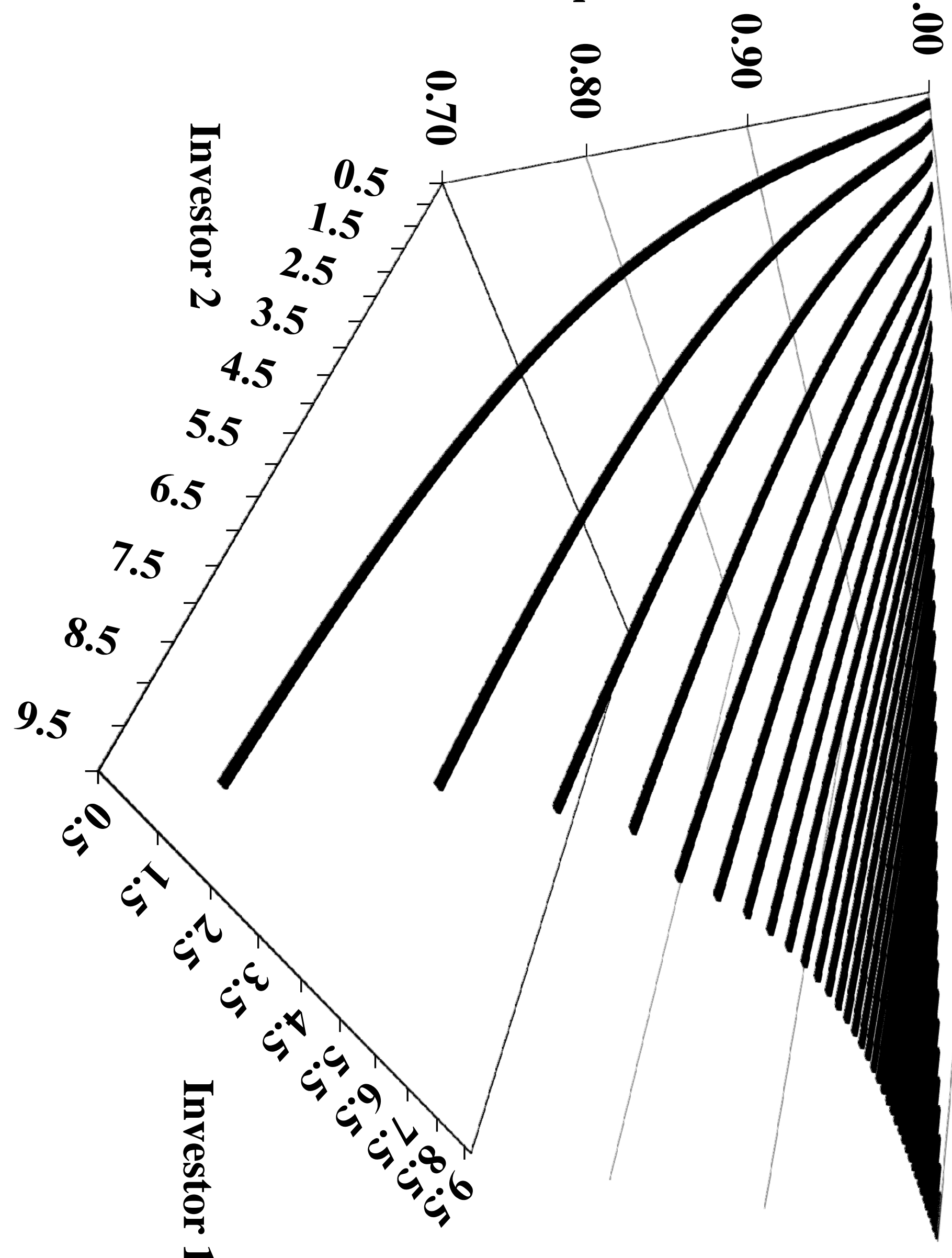




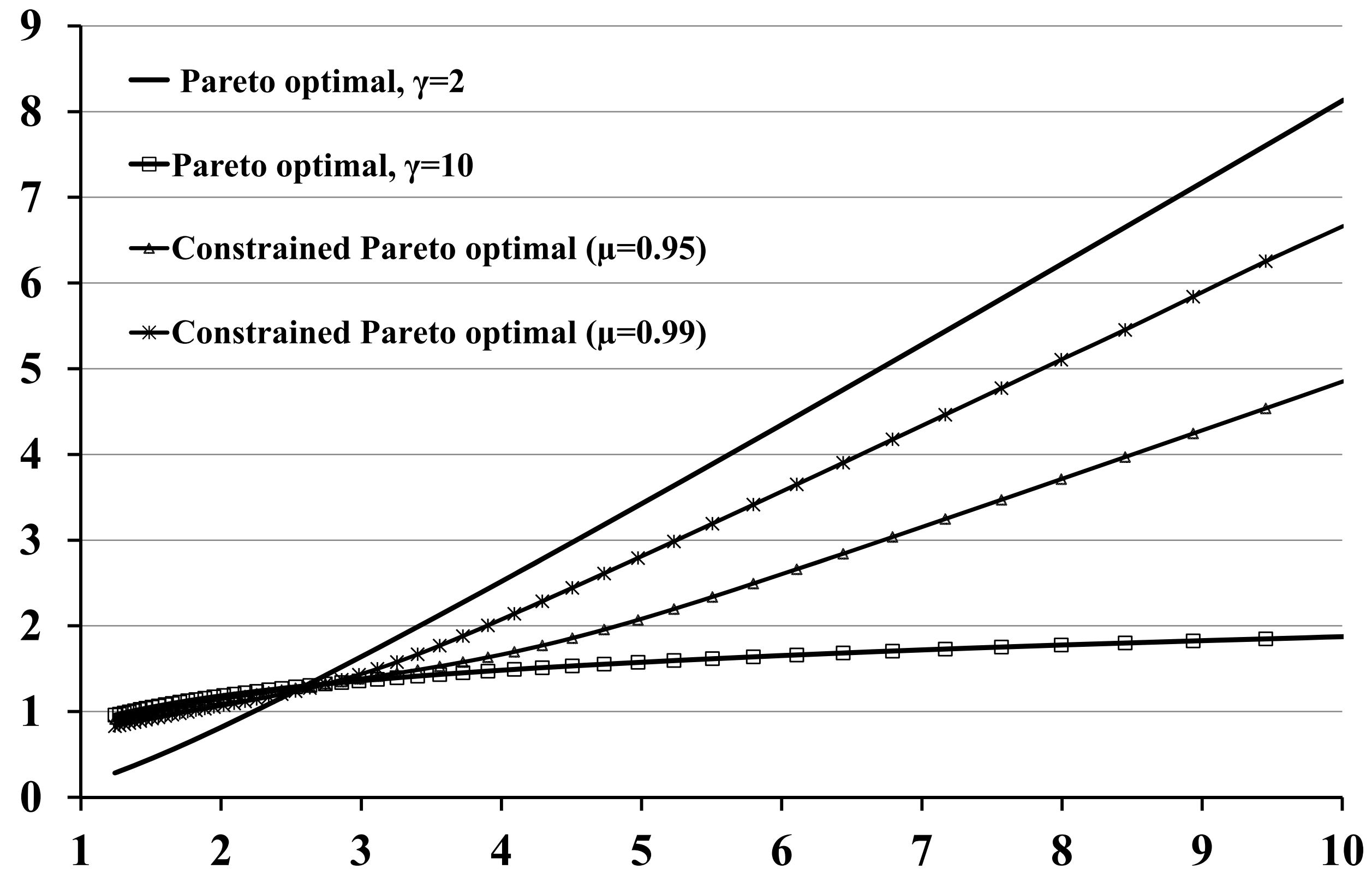




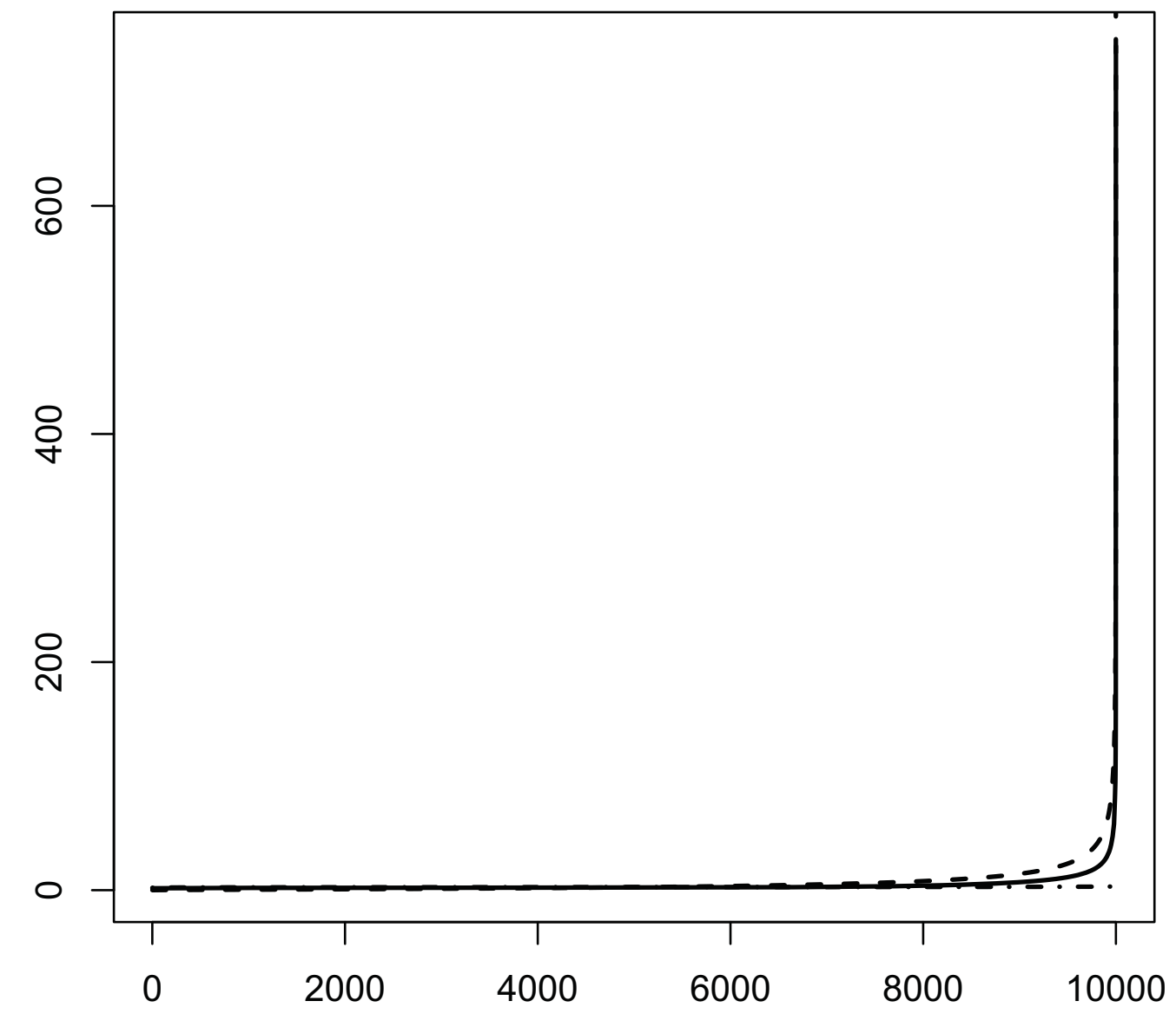




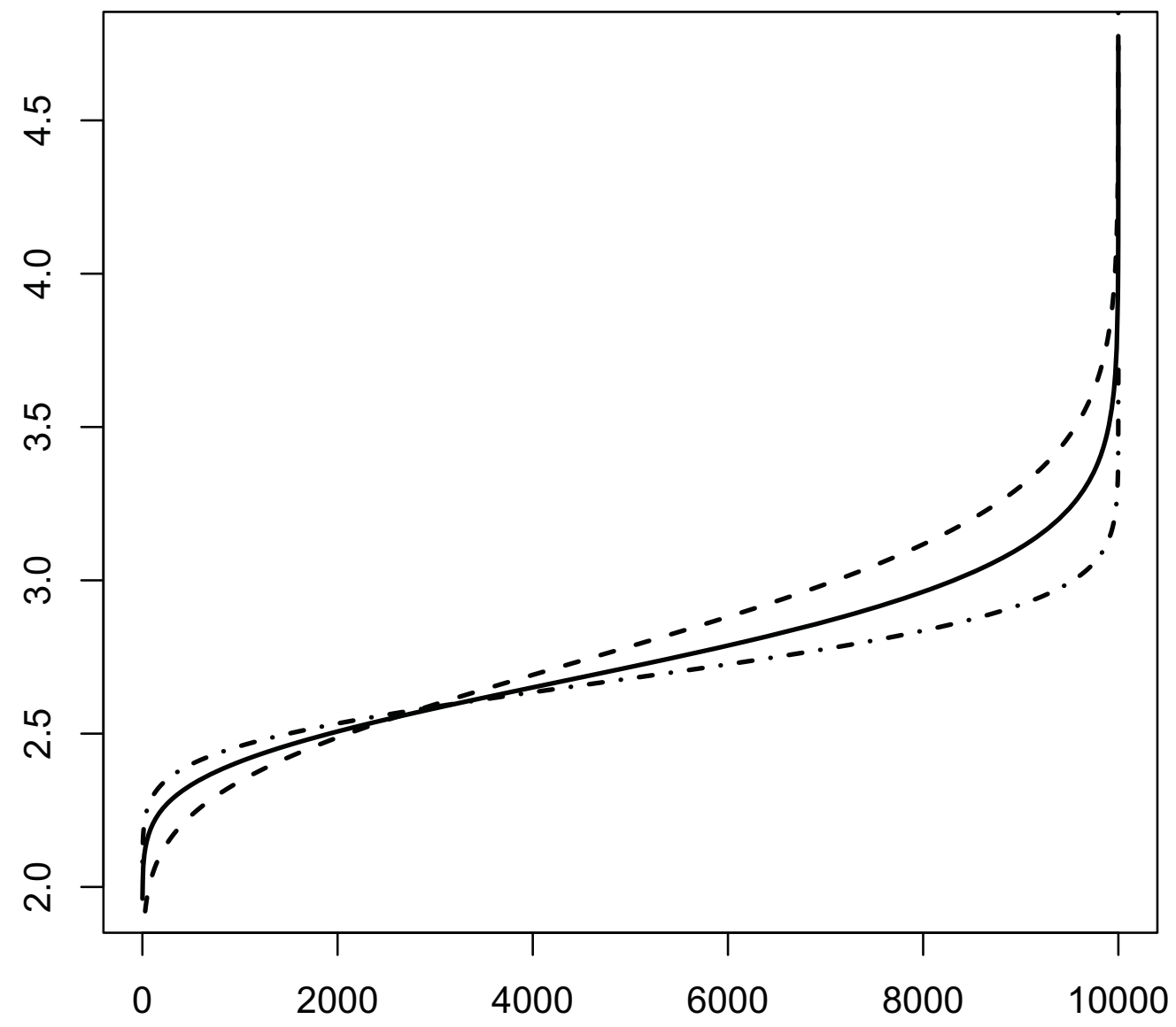




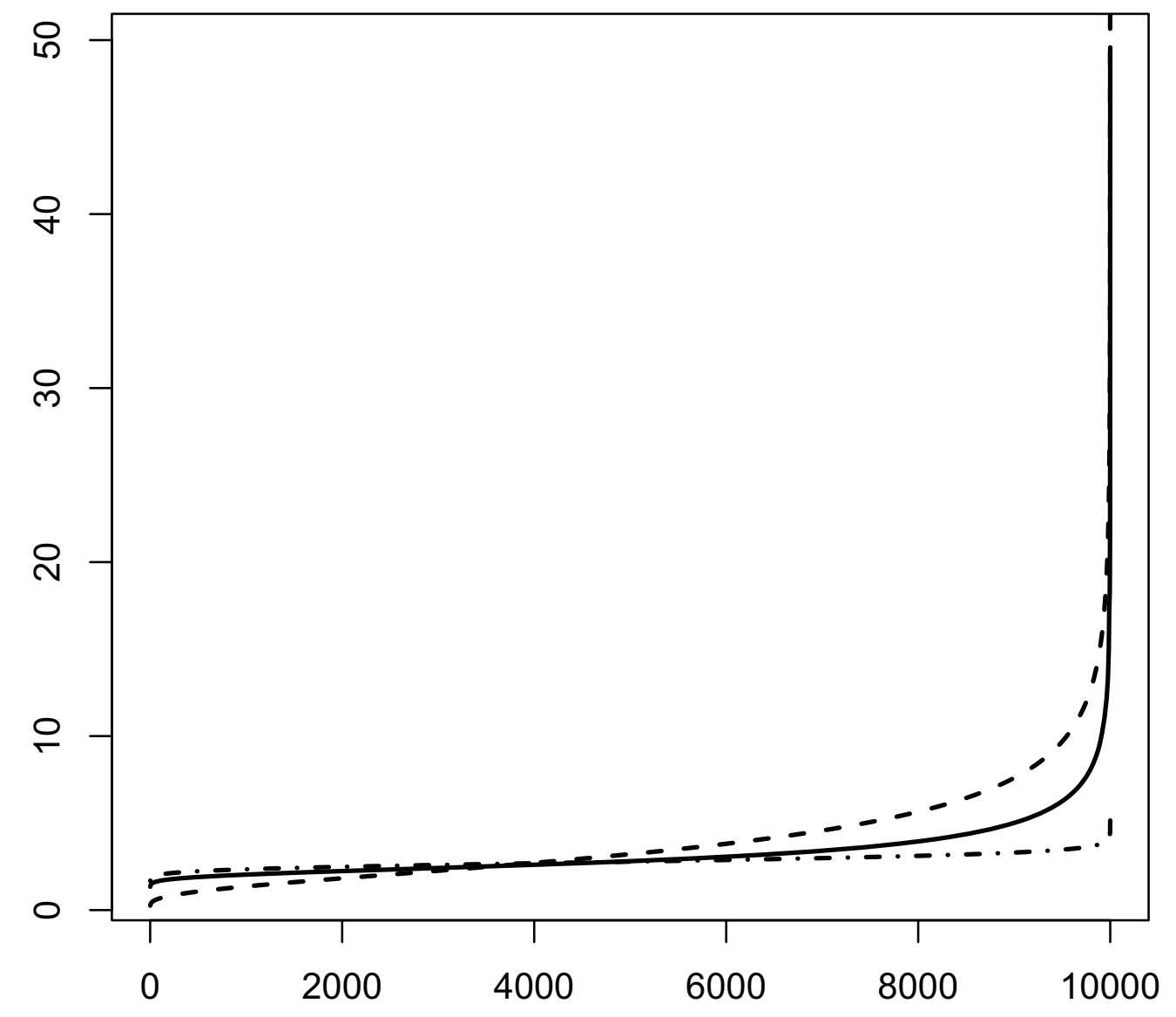

